\title{
Restoring brain cholesterol turnover improves autophagy and has therapeutic potential in mouse models of spinocerebellar ataxia
}

\author{
Clévio Nóbrega ${ }^{1,2,3,4} \cdot$ Liliana Mendonça $^{4} \cdot$ Adriana Marcelo $^{1,2} \cdot$ Antonin Lamazière $^{5} \cdot$ Sandra Tomé $^{4}$. \\ Gaetan Despres $^{5} \cdot$ Carlos A. Matos $^{1,2,4}$. Fatich Mechmet ${ }^{1,2} \cdot$ Dominique Langui $^{6}$. Wilfred den Dunnen ${ }^{7}$. \\ Luis Pereira de Almeida ${ }^{4,10}$. Nathalie Cartier ${ }^{8,9}$. Sandro Alves ${ }^{11}$ (1)
}

Received: 2 October 2018 / Revised: 4 April 2019 / Accepted: 20 April 2019 / Published online: 14 June 2019

(c) Springer-Verlag GmbH Germany, part of Springer Nature 2019

\begin{abstract}
Spinocerebellar ataxias (SCAs) are devastating neurodegenerative disorders for which no curative or preventive therapies are available. Deregulation of brain cholesterol metabolism and impaired brain cholesterol turnover have been associated with several neurodegenerative diseases. SCA3 or Machado-Joseph disease (MJD) is the most prevalent ataxia worldwide. We show that cholesterol 24-hydroxylase (CYP46A1), the key enzyme allowing efflux of brain cholesterol and activating brain cholesterol turnover, is decreased in cerebellar extracts from SCA3 patients and SCA3 mice. We investigated whether reinstating CYP46A1 expression would improve the disease phenotype of SCA3 mouse models. We show that administration of adeno-associated viral vectors encoding CYP46A1 to a lentiviral-based SCA3 mouse model reduces mutant ataxin-3 accumulation, which is a hallmark of SCA3, and preserves neuronal markers. In a transgenic SCA3 model with a severe motor phenotype we confirm that cerebellar delivery of AAVrh10-CYP46A1 is strongly neuroprotective in adult mice with established pathology. CYP46A1 significantly decreases ataxin-3 protein aggregation, alleviates motor impairments and improves SCA3-associated neuropathology. In particular, improvement in Purkinje cell number and reduction of cerebellar atrophy are observed in AAVrh10-CYP46A1-treated mice. Conversely, we show that knocking-down CYP46A1 in normal mouse brain impairs cholesterol metabolism, induces motor deficits and produces strong neurodegeneration with impairment of the endosomal-lysosomal pathway, a phenotype closely resembling that of SCA3. Remarkably, we demonstrate for the first time both in vitro, in a SCA3 cellular model, and in vivo, in mouse brain, that CYP46A1 activates autophagy, which is impaired in SCA3, leading to decreased mutant ataxin-3 deposition. More broadly, we show that the beneficial effect of CYP46A1 is also observed with mutant ataxin-2 aggregates. Altogether, our results confirm a pivotal role for CYP46A1 and brain cholesterol metabolism in neuronal function, pointing to a key contribution of the neuronal cholesterol pathway in mechanisms mediating clearance of aggregate-prone proteins. This study identifies CYP46A1 as a relevant therapeutic target not only for SCA3 but also for other SCAs.
\end{abstract}

Keywords Ataxia $\cdot$ Cholesterol metabolism $\cdot$ 24-Cholesterol hydroxylase $\cdot$ Autophagy $\cdot$ SCA animal models $\cdot$ SCA patients

Clévio Nóbrega and Liliana Mendonça contributed equally.

Luis Pereira de Almeida, Nathalie Cartier, Sandro Alves contributed equally.

Electronic supplementary material The online version of this article (https://doi.org/10.1007/s00401-019-02019-7) contains supplementary material, which is available to authorized users.

Extended author information available on the last page of the article

\section{Introduction}

Spinocerebellar ataxia type 3 (SCA3), also known as Machado-Joseph disease (MJD), is a progressive autosomal dominant inherited neurodegenerative disorder [25]. SCA3 is caused by a CAG-repeat expansion in the coding region of the $A T X N 3 / M J D 1$ gene, that results in an expanded polyglutamine (polyQ) tract in the coding region of the ataxin-3 protein [30]. This expansion is admitted to confer a toxic gain of function to the expanded ataxin-3 protein, which abnormally accumulates in neuronal cells and leads to neuronal demise 
$[52,62]$. This type of mutation is present in a family that comprises eight other inherited neurodegenerative disorders, including Huntington's disease (HD), dentatorubral-pallidoluysian atrophy (DRPLA), spinal and bulbar muscular atrophy (SBMA) and SCA types 1, 2, 6, 7 and 17 [37]. Clinically, SCA3 involves a range of progressive cerebellar dysfunctions, also associated with degeneration of the striatum, substantia nigra and cranial nerve motor nuclei $[4,25,31$, 56]. This neurodegenerative profile is translated in a broad spectrum of symptoms including ataxia, postural instability, oculomotor impairments, Parkinsonism, pyramidal signs and neuropathy $[15,57,61,62]$.

There is a wealth of evidence demonstrating that defects in brain cholesterol metabolism may contribute to diverse neurodegenerative disorders, such as Alzheimer's disease (AD), HD, Parkinson's disease (PD) and Niemann-Pick disease Type $\mathrm{C}[32,72]$. Cholesterol is critical for the physiology of neurons, all through development and during adult life, in the maintenance of synaptic activity [17, 38]. Brain cholesterol is almost exclusively synthesized in situ [23], since the blood-brain-barrier (BBB) prevents its passage from circulation and into the brain, and vice versa [11]. To be excreted from the brain, cholesterol is converted into 24S-hydroxycholesterol (24S-OHC), a molecule that dynamically crosses the BBB and reaches peripheral circulation, being eliminated in biliary acids [10]. This conversion is mediated by a key enzyme of brain cholesterol metabolism, termed cholesterol 24-hydroxylase (CYP46A1), a member of the cytochrome P450 family [41] mainly expressed in neurons [35]. CYP46A1 has been shown to play a major role in the turnover of brain cholesterol and in neuronal responses to stress $[10,66]$.

We have previously demonstrated that CYP46A1 levels are decreased in affected brain regions of patients and mouse models of HD and AD [13, 16]. In wild-type mice, CYP46A1 deficiency induces neuronal death associated with cognitive and motor dysfunctions [13, 24]. Conversely, CYP46A1-mediated gene therapy has been shown to rescue neurodegenerative hallmarks and behavior defects in rodent models of HD, efficiently clearing aggregates of mutant polyQ huntingtin.

We formulated the hypothesis that CYP46A1 could be beneficial in other polyQ diseases and particularly in SCA3. To our knowledge, the role of brain cholesterol metabolism in SCAs is poorly understood. We analyzed brain samples from SCA3 patients and evaluated the therapeutic benefits of CYP46A1 overexpression in two SCA3 mouse models: a lentiviral-based model and a transgenic model with severe established pathology. Our study (1) detected brain cholesterol metabolism dysfunction and CYP46A1 reduction in cerebellar samples of SCA3 patients; (2) demonstrated that adeno-associated virus (AAV)-mediated expression of CYP46A1 reduces the accumulation of ataxin-3 aggregates and alleviates SCA3-associated neuropathology, improving motor defects, in SCA3 models; (3) confirmed that a decrease in CYP46A1 expression and consequent brain cholesterol dysfunction leads to strong abnormalities of the endosomal-lysosomal compartment mimicking those observed in SCA3 disease; (4) importantly, proved that beneficial effects of CYP46A1 overexpression on SCA3 are associated with CYP46A1-mediated activation of autophagy, which is impaired in polyQ-SCAs $[1,46]$. Indeed, we show that CYP46A1 activates the autophagic pathway, which is compromised in SCA3, mitigating mutant ataxin-3 accumulation. This effect is further demonstrated for another SCA-causative protein, mutant ataxin-2, whose mutant ataxin-2 aggregates are reduced upon CYP46A1 overexpression. Taken together, our data suggest that AAVCYP46A1 brain delivery is a relevant therapeutic option to counteract human SCAs.

\section{Materials and methods}

\section{Post-mortem brain samples from SCA3 patients and control individuals}

Samples from the cerebellar cortex from five SCA3 patients (with expanded CAG repeats; five males, mean age at death: 56.4 years) and five age-matched control subjects (four males, one female, mean age at death: 66.2 years) were analyzed by western blot. In addition, post-mortem brains (in particular, the cerebellar cortex) from two patients with clinically, morphologically and genetically diagnosed SCA3/MJD along with the brains (cerebellar cortex) from three individuals with no evidence of neurological disease (negative controls) were used for immunohistochemistry. All brains were obtained from the University Medical Centre Groningen, the Netherlands. Autopsies were carried out by accredited pathologists, after informed consent was obtained from all patients in accordance with the medical ethical committee of the University Medical Centre Groningen, the Netherlands, where the autopsies were performed. All SCA3 patients suffered from gait, stance and limb ataxia, dysarthria, dysphagia and a variety of oculomotor dysfunctions. Genetic diagnosis was carried out in all SCA3 patients by genotyping the DNA extracted from peripheral lymphocytes with polymorphic dinucleotide repeat sequences that flank the specific $A T X N 3 / M J D 1$ gene loci [30]. The pathologically expanded CAG repeat number varied between 64 and 81 in the SCA3 patients analyzed (see Supplementary table I/ Online Resource 1). 


\section{Lentiviral vectors production}

Lentiviral vectors encoding human mutant ataxin-3 (LVPGK-Atx-3 72Q) were produced in HEK 293T cells using a four-plasmid system, as previously described [4].

\section{AAV plasmid design and vector production}

AAV vectors were produced and purified by Atlantic Gene therapies (INSERM U1089, Nantes, France). Vector production has been described elsewhere [28]. The viral constructs for AAVrh10-GFP and AAVrh10-CYP46A1 contained an expression cassette consisting of either the cDNA encoding green fluorescent protein (GFP) or the human CYP46A1 protein, driven by a CMV early enhancer/chicken $\beta$-actin (CAG) synthetic promoter (CAG) surrounded by inverted terminal repeat (ITR) sequences of AAV. The AAVrh10-CAG-GFP vector was also generated and was used as control. The viral constructs AAV5-shScramble-GFP and AAV5-shCYP46A1GFP contained an expression cassette consisting of either short-hairpin (sh) CYP46A1 (shCyp46a1) or a scrambled sequence (shScramble) driven by a PU6 promoter, and a GFP reporter gene, driven by the phosphoglycerate kinase 1 (PGK1) promoter, in the AAV5 vector [13, 24].

\section{Animals}

Five-week-old C57BL/6J mice $(n=8)$ obtained from Charles River Laboratories (Spain), as well as 6-week-old or 3-month-old transgenic SCA3 Q69 mice $(n=25)$ and agematched wild-type littermates $(n=10)[53,71]$ bred in-house were used in the evaluation of AAV-CYP46A1 efficacy in SCA mouse models. SCA3 transgenic mice (C57BL/6 background) overexpress $\mathrm{N}$-terminally truncated human ATXN3, containing $69 \mathrm{CAG}$ repeats and an $\mathrm{N}$-terminal haemagglutinin (HA) tag epitope, driven specifically in cerebellar Purkinje cells by the L 7 promoter [53]. This truncated ATXN3 fragment lacks the $286 \mathrm{~N}$-terminal amino acids that contain the deubiquitinase domain (Josephin domain) and two ubiquitin-interacting motifs (UIMs). Therefore, the truncated protein consists of a 69 polyQ tract with only 4 and 42 amino acids at its $\mathrm{N}$-terminus and $\mathrm{C}$-terminus, respectively. In addition, 16 2-month-old $\mathrm{C} 57 \mathrm{Bl} / 6$ mice were injected in the striatum with an AAV5 vector containing an expression cassette consisting of either short-hairpin (sh) CYP46A1 (AAV5-shCyp46a1) $(n=11)$ or a scrambled sequence (AAV5-shScramble) $(n=11)$. Mice were housed in a temperature-controlled room and maintained on a $12 \mathrm{~h}$ light/dark cycle. Food and water were available ad libitum. The experiments were carried out in accordance with the European Community Council directive (2010/63/EU) for the care and use of laboratory animals. The researchers received adequate training (FELASA certified course) and certification to perform the experiments from Portuguese authorities (Direção-Geral de Alimentação e Veterinária).

\section{Stereotactic injections in the mouse brain}

Five-week-old C57BL6/J mice were anaesthetized with Avertin (14 ml g/1 and $250 \mathrm{mg} \mathrm{kg} / 1$, intraperitoneally). For each animal, $400.000 \mathrm{ng}$ of $\mathrm{p} 24$ antigen of lentiviral vectors encoding ATXN3 72Q (MUT ATXN3) and $1.10^{9} \mathrm{vg} / \mu \mathrm{l}$ of AAVrh10 encoding CYP46A1 ( $n=4$; bilateral injection) or GFP (control; $n=4$; bilateral injection) in a final volume of $2 \mu \mathrm{l}$ were stereotactically co-injected $(0.2 \mu \mathrm{l} / \mathrm{min})$ into the striatum in the following coordinates: anteroposterior: $+0.6 \mathrm{~mm}$, lateral: $\pm 1.8 \mathrm{~mm}$, ventral: $-3.3 \mathrm{~mm}$ and tooth bar: 0 .

In addition, 16 8-week-old C57BL6/J mice were injected bilaterally into the striatum with an AAV5-shCYP46A1 or an AAV5-shScramble $\left(3 \times 10^{9} \mathrm{vg}\right.$ in $\left.2 \mu \mathrm{l}\right)$. These mice were injected using the same striatum coordinates described above.

For cerebellar injections, 6-week-old transgenic SCA3 Q69 mice $(n=25)$ and age-matched wild-type littermates $(n=10)$ were injected into the cerebellar vermis with AAVrh10-CYP46A1 or AAVrh10-GFP $\left(2 \mu \mathrm{l} ; 3.10^{9} \mathrm{vg}\right)$ at $0.25 \mu \mathrm{l} / \mathrm{min}$ at the coordinates: $-1.6 \mathrm{~mm}$ rostral to lambda, $0 \mathrm{~mm}$ midline, and $-1 \mathrm{~mm}$ ventral to the skull surface, with the mouth bar set at -3.3 .

After injection, the syringe needle was left in place for an additional $5 \mathrm{~min}$ before being slowly raised. The skin was closed using a 6-0 Prolene ${ }^{\circledR}$ suture (Ethicon, Johnson and Johnson, Brussels, Belgium).

\section{Behavioral tests in transgenic SCA3 Q69 mice}

To evaluate the impact of AAVrh10-CYP46A1 overexpression on the motor phenotype, transgenic SCA3 Q69 mice were injected with AAVrh10-CYP46A1 $(n=10)$ or either AAVrh10-GFP (control; $n=9$ ); in addition, four littermate wild-type C57BL/6 animals were used. Mice were trained on a battery of motor tests, starting at 5-6 weeks of age, and performed every $2 / 4$ weeks, from week 2 after injection, until 10 weeks post-injection. Procedures were carried out by an experienced operator, blind to condition. All tests were performed in the same dark room after $30 \mathrm{~min}$ of acclimatization.

\section{Rotarod test}

Motor coordination and balance were evaluated with a Rotarod apparatus (Letica Scientific Instruments). Mice were placed on the Rotarod apparatus, at a constant speed ( $5 \mathrm{rpm}$ ) for a maximum of $5 \mathrm{~min}$ and at an accelerating speed (4-40 rpm, in $5 \mathrm{~min}$ ), and the latency to fall was 
recorded. Mice performed three trials for each time point with a 15-20 min rest between trials. The mean latency to fall off the Rotarod in the three trials was used for analysis.

\section{Footprint pattern analysis}

See Supplementary Materials and Methods (Online Resource 2).

\section{Mouse brain sample processing}

Non-injected transgenic SCA3 Q69 mice $(n=6)$ and agematched wild-type littermates $(n=6)$ were sacrificed at 3 months of age. After an overdose of sodium pentobarbital, animals were perfused transcardially with ice-cold PBS $0.1 \mathrm{M}$ before brain extraction. For western blot processing, the cerebellum was dissected and then homogenized in a lysis buffer (TBS, $\mathrm{NaCl} 150 \mathrm{mM}$ and Triton 1\%) containing phosphatase (Pierce) and protease (Roche) inhibitors. After centrifugation (20 min, 13,000 rpm, $4{ }^{\circ} \mathrm{C}$ ), the supernatant was collected. Lysate aliquots ( $3 \mathrm{mg}$ of protein $/ \mathrm{ml}$ ) were stored at $-80{ }^{\circ} \mathrm{C}$ until use. The other half of the cerebellum was conserved at $-80{ }^{\circ} \mathrm{C}$ until gas chromatography-mass spectrometry (GC-MS) analysis.

C57BL/6 mice ( $n=11 /$ group) injected with AAV5shCYP46A1 or AAV5-shScramble were perfused transcardially with ice-cold PBS $0.1 \mathrm{M}$ before striatum extraction. Half of the brain was post-fixed in $4 \%$ paraformaldehyde (PFA) (Fluka, Sigma) in 0.1 M PBS for 3 days and cryoprotected by incubation in a 20\% sucrose/0.1 M PBS solution. Coronal brain sections $(25 \mu \mathrm{m})$ were cut on a freezing microtome (Leica, Wetzlar, Germany), collected serially, and stored at $4{ }^{\circ} \mathrm{C}$, free-floating in $0.02 \%$ sodium azide PBS until immunohistochemical processing. The striatum of the contralateral hemisphere was dissected and conserved at $-80{ }^{\circ} \mathrm{C}$ until GC-MS analysis. For western blot analysis, animals were killed through sodium pentobarbital overdose without transcardial perfusion. Brains were removed and dissected fresh for collection of striatal punches using a Harris Uni-Core pen, with a $2.0 \mathrm{~mm}$ diameter (Ted Pella, Inc.). Brain punches samples were then stored at $-80{ }^{\circ} \mathrm{C}$ until posterior processing.

The brains of transgenic SCA3 Q69 mice injected with AAVrh10-CYP46A1 or AAVrh10-GFP and age-matched wild-type mice were removed, post-fixed in $4 \%$ PFA for $24 \mathrm{~h}$ and cryoprotected by incubation in $25 \%$ sucrose/phosphate buffer for $48 \mathrm{~h}$. Subsequently, brains were frozen and sagittally sectioned ( $40 \mu \mathrm{m}$ sections) using a cryostat (LEICA CM3050 S). Brain sections were collected and stored in 48-well trays, free-floating in $0.1 \mathrm{M}$ phosphate buffer solution supplemented with $0.05 \mathrm{mM}$ sodium azide and stored at $4{ }^{\circ} \mathrm{C}$ until further usage.

\section{Cholesterol and oxysterol measurements}

Cholesterol and oxysterol analysis followed the 'gold standard' method [26] to minimize the formation of autoxidation artifacts. Briefly, mouse striatal tissue samples were weighed and homogenized with a TissueLyser II apparatus (Qiagen) in a $500 \mu \mathrm{l}$ solution containing butylated hydroxytoluene (BHT, $50 \mu \mathrm{g} / \mathrm{ml}$ ) and EDTA $(0.5 \mathrm{M})$. At this point, a mix of internal standards was added [epicoprostanol, ${ }^{2} \mathrm{H}_{7}$-7-lathosterol, ${ }^{2} \mathrm{H}_{6}$-desmosterol, ${ }^{2} \mathrm{H}_{6}$-lanosterol and ${ }^{2} \mathrm{H}_{7}-24(\mathrm{R} / \mathrm{S})$-hydroxycholesterol] (Avanti Polar Lipids). Alkaline hydrolysis was performed under Ar using $0.35 \mathrm{M}$ ethanolic $\mathrm{KOH}$ for $2 \mathrm{~h}$ at room temperature. After neutralization of the solution with phosphoric acid, sterols were extracted in chloroform. The lower phase was collected, dried under a stream of nitrogen and the residue was dissolved in toluene. Oxysterols were then separated from the cholesterol and its precursors on a $100 \mathrm{mg}$ Isolute silica cartridge (Biotage); cholesterol was eluted in 0.5\% propan-2-ol in hexane followed by oxysterols in 30\% propan-2-ol in hexane. The sterol and oxysterol fractions were independently silylated with Regisil ${ }^{\circledR}+10 \%$ TMCS [bis(trimethylsilyl) trifluoro-acetamide $+10 \%$ trimethylchlorosilane] (Regis technologies) as described previously [19]. The trimethylsilylether derivatives of sterols and oxysterols were separated by gas chromatography (Hewlett-Packard 6890 series) in a medium polarity capillary column RTX-65 (65\% diphenyl $35 \%$ dimethyl polysiloxane, length $30 \mathrm{~m}$, diameter $0.32 \mathrm{~mm}$, film thickness $0.25 \mu \mathrm{m}$; Restesk). The mass spectrometer (Agilent 5975 inert XL) in series with the gas chromatography was set up for detection of positive ions. Ions were produced in the electron impact mode at $70 \mathrm{eV}$. They were identified by the fragmentogram in the scanning mode and quantified by selective monitoring of the specific ions after normalization and calibration with the appropriate internal and external standards [epicoprostanol $\mathrm{m} / z, 370,{ }^{2} \mathrm{H}_{7}-7$ lathosterol $\mathrm{m} / \mathrm{z}, 465,{ }^{2} \mathrm{H}_{6}$-desmosterol $\mathrm{m} / z, 358,{ }^{2} \mathrm{H}_{6}$-lanosterol $m / z$ 504, ${ }^{2} \mathrm{H}_{7}-24(\mathrm{R} / \mathrm{S})$-hydroxycholesterol $\mathrm{m} / z$ 413, cholesterol $\mathrm{m} / \mathrm{z}, 329$, 7-lathosterol $\mathrm{m} / \mathrm{z}$ 458, 7-dehydrocholesterol $\mathrm{m} / \mathrm{z} 325,8$-dehydrocholesterol $\mathrm{m} / \mathrm{z} 325$, desmosterol $\mathrm{m} / \mathrm{z} 351$, lanosterol $\mathrm{m} / z 393$ and 24(R/S)-hydroxycholesterol $\mathrm{m} / \mathrm{z} 413$ ].

\section{Western blot}

Total protein concentrations were determined using the BCA kit (Pierce). Equal amounts of total protein extract $(30 \mu \mathrm{g})$ were electrophoretically separated using SDS-PAGE in $4-12 \%$ Bis-Tris gels $\left(\right.$ NuPAGE$^{\circledR}$ Novex Bis-tris midi gel 15 or 26 wells, Life Technologies, Carlsbad, USA) and transferred to nitrocellulose membranes. Blocked membranes (5\% non-fat dry milk in TBS-0.1\% Tween-20) were incubated with primary antibodies overnight at $4{ }^{\circ} \mathrm{C}$ and washed three times with TBS- $-0.1 \%$ 
Table 1 Antibodies used in western-blot (WB) and immunohistochemical (IHC) analyses

\begin{tabular}{llll}
\hline Primary antibodies & Source & WB & IHC/IF \\
\hline $\begin{array}{l}\text { Mouse anti-ATXN3 (clone } \\
\text { 1H9) }\end{array}$ & Millipore & $1: 1000$ & $1: 2000$ \\
$\begin{array}{l}\text { Mouse anti-hemagglutinin } \\
\text { tag }\end{array}$ & Covance & $1: 4000$ & $1: 5000$ \\
Rabbit anti-ubiquitin & Enzo life technologies & - & $1: 300$ \\
Rabbit anti-calbindin & Chemicon/Millipore & $1: 2000$ & $1: 1000$ \\
Rabbit anti-CYP46A1 & Abcam & $1: 2000$ & $1: 3000$ \\
Rabbit anti-DARPP-32 & Millipore & - & $1: 2000$ \\
Rabbit anti-GFP & Abcam & $1: 2000$ & $1: 5000$ \\
Anti-Cathepsin-D & Abcam & - & $1: 3000$ \\
Anti-LAMP-1 clone 1D4B & Abcam & - & $1: 500$ \\
Anti-Rab5 & Abcam & - & $1: 2000$ \\
Anti-Rab7 & Abcam & - & $1: 2000$ \\
Rabbit anti-LC3B & Cell signaling & $1: 1000$ & - \\
Rabbit anti-SQSTM1/p62 & Cell signaling & $1: 1000$ & - \\
Mouse anti-GAPDH & Abcam & $1: 4000$ & - \\
Mouse anti-actin & Sigma & $1: 5000$ & - \\
Rabbit anti-Iba1 & Wako & - & $1: 3000$ \\
Rabbit anti-GFAP & Dako & - & $1: 5000$ \\
Rabbit anti-cleaved cas- & Cell signaling & - & $1: 1000$ \\
pase-3 & & & \\
Mouse anti-NeuN & Millipore & - & $1: 500$ \\
\hline
\end{tabular}

Tween-20 (T-BST) for $10 \mathrm{~min}$. Membranes were then labeled with secondary IgG-HRP antibodies raised against each corresponding primary antibody. After three washes with T-BST, the membranes were incubated with ECL chemiluminescent reagent (Clarity Western ECL substrate; GE Healthcare, Little Chalfont, UK) according to the instructions of the supplier. Peroxydase activity was detected with a Fusion TX7 camera system (Fisher Scientific). Quantification was made by densitometric analysis with the Quantity One 1D image analysis software (version 4.4; Biorad, Hercules, CA, USA). The optical densities were normalized with respect to a "standard protein" (GAPDH/actin). For each sample, a partition ratio was calculated and normalized with respect to the sample with the highest value defined as 1 .

\section{Primary antibodies}

Antibodies used in western blot (WB) and immunohistochemical (IHC) analyses are described in Table 1.

\section{Immunostaining}

The immunohistochemical procedure was initiated by quenching endogenous peroxidase activity by incubating free-floating sections in phenylhydrazine for $25 \mathrm{~min}$ at $37^{\circ} \mathrm{C}$. After three washes, slices were blocked in PBS/0.1\% Triton X-100 containing 10\% normal goat serum (NGS, Gibco) for $1 \mathrm{~h}$ at RT. The sections were then incubated with the respective primary antibodies, overnight at $4{ }^{\circ} \mathrm{C}$. After three washings, the sections were incubated with the corresponding biotinylated secondary antibody (1:250; Vector Laboratories Inc., CA, USA) diluted in PBS/0.1\% Triton X-100 and 10\% NGS for $2 \mathrm{~h}$ at RT. After three washes, bound antibodies were visualized by the $\mathrm{ABC}$ amplification system (Vectastain ABC kit, Vector Laboratories, West Grove, USA) and 3,3'-diaminobenzidine tetrahydrochloride (peroxidase substrate kit, DAB, Vector Laboratories, CA, USA) as the substrate. The sections were mounted, dehydrated by passing twice through ethanol and toluol solutions, and coverslipped with Eukitt ${ }^{\circledR}(\mathrm{O}$. Kindler GmbH \& CO, Freiburg, Germany).

For immunofluorescence, slices were washed with PBS $0.1 \mathrm{M}$ and permeabilized in PBS-Triton $0.1 \%$ before blocking in PBS-Triton $0.1 \%$ containing 5\% NGS for $1 \mathrm{~h}$. Sections were then incubated with the respective primary antibodies, overnight at $4{ }^{\circ} \mathrm{C}$. After three successive washes brain slices were incubated for $2 \mathrm{~h}$ at RT with fluorescent secondary Alexa Fluor-conjugated antibodies (Invitrogen). Sections were then incubated with DAPI solution (1:5000) for $10 \mathrm{~min}$ at room temperature, washed and mounted in FluorSaveTM Reagent (Calbiochem) on microscope slides and conserved at $4{ }^{\circ} \mathrm{C}$.

\section{Cresyl violet staining}

Cerebellar sections were stained with cresyl violet for $2 \mathrm{~min}$, differentiated in acetate buffer $\mathrm{pH} 3.8-4$ (2.72\% sodium acetate and $1.2 \%$ acetic acid; $1: 4 \mathrm{v} / \mathrm{v}$ ), dehydrated by passing twice through ethanol and toluol solutions, and mounted with Eukitt (O. Kindler GmbH \& CO. Freiburg, Germany).

\section{Quantitative analysis of ataxin-3 and ubiquitin-positive aggregates and DARPP-32 depletion in immunohistochemically labeled brain slices from the LV-based model of SCA3}

Brain slices were analyzed and imaged at RT with an Axiovert $200 \mathrm{M}$ microscope (CCD color digital Axiocam HRc camera; Carl Zeiss), at $5 \times$ (air objective, Fluar, $0.25 \mathrm{NA}$ ) and $20 \times$ magnifications (air objective, LD-PlanNeofluar, 0.4 NA). Composite images of complete aggregate-containing or DARPP-32-depleted regions were automatically acquired using the MozaiX function of the AxioVision software. Photographs for comparison were taken under identical conditions of image acquisition, and all adjustments of brightness and contrast were applied uniformly to all images. Quantification of ataxin-3/ubiquitin-positive aggregates 
and determination of the area of the DARPP-32 depletion was made in 7-8 slices for each mouse, spread over the anterior-posterior extent of the striatum and separated by $200 \mu \mathrm{m}$. Counting of ataxin-3/ubiquitin-positive aggregates was performed on $20 \times$-magnified images for each hemisphere using ImageJ (National Institutes of Health). After manually thresholding images so that the recognizable ataxin-3-positive accumulations were included in the examination, particles $>3 \mu \mathrm{m}^{2}$ were automatically analyzed and counted (scale: 2 pixels $/ \mu \mathrm{m}$ ), and the estimative calculation of the total number of aggregates in the entire striatum was performed as previously described [2,3]. The extent of mutant ataxin-3-induced lesions in the striatum was analyzed by digitizing 6-8 DARPP-32-stained sections per animal ( $200 \mu \mathrm{m}$ between $25 \mu \mathrm{m}$ thickness sections), selected to obtain a complete rostro-caudal sampling of the striatum, with a slide scanner and by quantifying the area of the lesion with a semiautomated image-analysis program (ImageJ software, NIH, MD, USA), to manually define the regions with decreased antibody reactivity and quantify their area (scale: 0.5 pixels $/ \mu \mathrm{m})$. The volume was then estimated using the following formula: volume $=d(a 1+a 2+a 3+\cdots)$, where $d$ is the distance between serial sections ( $200 \mu \mathrm{m})$, and $a 1, a 2$, $a 3$, etc., are DARPP-32-depleted areas for individual serial sections [4]. The average gray value of all pixels measured in the lesion area was recorded for each depleted area. Results are presented as the calculated value for the volume of the DARPP-32-depleted region for each animal. In all cases, imaging, measurements, and quantifications were performed blindly to the treatment.

\section{Quantitative analysis of cresyl violet-stained and immunohistochemically labeled brain slices from SCA3 Q69 transgenic mice}

The cerebellar volume was blindly assessed by scanning the area per lobe in eight sections stained with cresyl violet, covering the full extent of the cerebellum. The cerebellar volume was obtained by multiplying the total area by the distance between serial sections $(320 \mu \mathrm{m})$.

Quantification of granular and molecular layers' size was made over four cresyl violet-stained sections spread over the anterior-posterior extent of the cerebellum in a blind fashion using a $20 \times$ magnification objective. For each section, the size of the layers was assessed blindly per lobe in 3-5 different measurements (depending on the lobule size).

For quantification of Purkinje cells, fluorescence images of eight sagittal sections covering the entire cerebellum, stained with an anti-calbindin antibody and DAPI, were acquired with a Zeiss Axio Imager Z2 microscope using a $20 \times$ objective. The number of Purkinje cells was manually counted for each section and for each cerebellar lobule, in a blind fashion. Data are represented as mean value of the number of Purkinje cells in the entire cerebellum or per lobule.

The number of ubiquitin-positive aggregates was assessed in lobules IX and X automatically using the Cell Profiler software, from images of eight sagittal sections covering the entire cerebellum, stained with an anti-ubiquitin antibody and DAPI. Images were acquired with a Zeiss Axio Imager Z2 microscope using a $20 \times$ objective. The analysis modules applied were the same for all the animals and images. Data are represented as the average number of ubiquitin aggregates per section.

\section{Neuroblastoma cell culture and transfection}

Mouse neuroblastoma cell line (N2a cells) obtained from the American Type Culture Collection cell biology bank (CCL-131) was maintained in Dulbecco's modified Eagle's medium (DMEM) supplemented with $10 \%$ fetal bovine serum (FBS), $100 \mathrm{U} / \mathrm{ml}$ penicillin and $100 \mathrm{mg} / \mathrm{ml}$ streptomycin (Gibco) at $37{ }^{\circ} \mathrm{C}$ in $5 \% \mathrm{CO}_{2} /$ air atmosphere. Human full-length mutant ataxin-3 with 84 glutamines (pEGFPAtx3Q84) [18] and a truncated form of mutant ataxin-2 with 104 glutamines based on the pEGFP-Atx2Q104 plasmid [29] were transfected into Neuro2A cells using polyethylenimine (PEI) and a total concentration of $0.5 \mathrm{mg}$ of each DNA construct was used. Cells were lysed for western blot processing or fixed for fluorescence microscopy analysis, $48 \mathrm{~h}$ post-transfection.

\section{Autophagic flux measurement}

The autophagic flux was measured through the LC3B turnover assay, which is used to quantify the amount of LC3B-II that is delivered to the lysosomes [5]. For this purpose, the levels of LC3B-II were measured by western blot in the presence and absence of chloroquine (ChQ, SIGMA, $100 \mu \mathrm{M}$ ), an inhibitor of autolysosomal degradation. The autophagic flux was calculated by subtracting the densitometric value of LC3B-II in samples without chloroquine (LC3B-II - ChQ) from the corresponding sample treated with chloroquine (LC3B-II + ChQ), for each condition.

\section{Ubiquitin-proteasome system (UPS) inhibition in neuroblastoma cells}

Six hour prior to collection (and $42 \mathrm{~h}$ post-transfection), transfected N2a cells were treated with MG-132 (SigmaAldrich, $5 \mu \mathrm{M}$ ), an ubiquitin-proteasome system (UPS) inhibitor. After this treatment N2a cells were collected for western blot and immunofluorescence analyses. 


\section{Quantification of mutant ataxin-3 and ataxin-2 aggregates in cell cultures}

Transfected cells were washed with PBS and fixed with 4\% paraformaldehyde (Sigma) at room temperature for $15 \mathrm{~min}$. Cells were then washed with PBS and nuclei were stained with DAPI (1:5000; Applichem) for $5 \mathrm{~min}$ at RT. After a washing step, the coverslips were mounted on microscope slides using Mowiol. Images were acquired with a $40 \times$ objective using a Zeiss Axio Imager Z2. For each condition at least four independent microscopy fields were acquired for a total of 100 transfected cells for each of the independent experiments. For each field, the number of cells with mutant ataxin-3 or ataxin-2 aggregates was counted.

\section{Statistics}

Statistical analysis was performed using the Mann-Whitney test and paired or unpaired Student's $t$ test or ANOVA for multiple comparisons, two-way ANOVA analysis with Bonferroni post-test, one-way ANOVA analysis with Bonferroni post-test or unpaired Student's $t$ test. Results are expressed as mean \pm SEM. Significant thresholds were set at $P<0.05$, $P<0.01, P<0.001$ and $P<0.0001$, as defined in the text. All analyses were performed using GraphPad Prism (GraphPad Software, La Jolla, USA).

\section{Results}

\section{Cholesterol metabolism pathway is impaired in the cerebellum of SCA 3 patients and SCA3 transgenic mice}

There are no studies addressing cholesterol metabolism dysregulation in SCAs associated with polyQ expansion. To investigate the possibility of such dysregulation taking place, we started by assessing CYP46A1 protein levels in post-mortem SCA3 patient cerebellum, in particular at the cerebellar cortex, a brain region affected in SCA3 [25]. Analyzing samples from five SCA3/MJD patients and five age-matched healthy individuals, we observed a statistically significant decrease (44\%) in CYP46A1 protein levels in the cerebellar cortex of SCA3 patients relatively to controls (Student's $t$ test; $P=0.0397$ ) (Fig. 1a, b). Since CYP46A1 is present in neurons we evaluated the levels of the calbindin-D-28K protein and demonstrated a $34 \%$ decrease (Student's $t$ test; $P=0.04$ ) in SCA3 patients compared to controls (Fig. 1a, b). Interestingly, we did not find major alterations in CYP46A1 levels in SCA3 patient 3, which also had increased levels of calbindin relatively to the remaining SCA3 patients. These data suggest that CYP46A1 levels may be correlated with the expression of neuronal markers
(Student's $t$ test; $P=0.04$ ). Of note, immunohistochemistry revealed that CYP46A1 levels were increased in the soma of Purkinje cells (PCs) from control individuals relatively to PCs from SCA3 patients, which presented ataxin-3-positive deposits in the nucleus, absent in control PCs (Fig. 1c).

We next evaluated the levels of CYP46A1 in brain samples from six SCA3/MJD transgenic mice expressing the N-terminal truncated human ataxin-3 with 69 CAG repeats, and six age-matched control littermates by western blot. In particular, we analyzed CYP46A1 protein levels in the cerebellum, since in this mouse model mutant human ataxin-3 is mostly expressed in PCs [53] (Fig. 1d). Results demonstrated a $\sim 20 \%$ reduction in CYP46A1 protein levels (Student's $t$ test; $P=0.0013$ ) in whole cerebellar extracts from SCA3 Q69 mice $(n=6)$ relatively to controls $(n=6)$ (Fig. 1d, e), while the levels of the neuronal markers NSE and NeuN remained unchanged (data not shown). Nevertheless, a statistical significant decrease in the levels of calbindin protein, mostly present in PCs (Fig. 1d, f) was observed. Immunofluorescence studies also revealed decreased CYP46A1 immunoreactivity in cerebellar sections from SCA3 Q69 mice compared to wild-type littermates (Suppl Fig. 1; Online Resource 3).

To determine the impact of CYP46A1 deficiency in SCA3, we assessed cholesterol metabolism by performing quantitative measurements of cholesterol and $24 \mathrm{~S}-\mathrm{OHC}$ by gas chromatography-mass spectrometry (GC-MS) analysis of cerebellar extracts from SCA3 Q69 mice $(n=6)$ and age-matched littermates $(n=6)$. We observed a statistically significant reduction $(21.3 \%)$ in the levels of $24 \mathrm{~S}-\mathrm{OHC}$ in SCA3 Q69 mice $(20.62 \pm 1.14 \mathrm{ng} / \mathrm{mg} ; n=6)$ relatively to wild-type littermates $(26.19 \pm 0.78 \mathrm{ng} / \mathrm{mg} ; n=6$, Student's $t$ test, $P=0.002$ ) (Fig. $1 \mathrm{~g}$ ). No statistically significant differences were observed for the cerebellar cholesterol content among SCA3 mice and wild-type mice (Fig. 1h). No statistically significant differences were found for the oxysterols $25 \mathrm{~S}-\mathrm{OHC}$ and $27 \mathrm{~S}-\mathrm{OHC}$ (data not shown). Overall, these results demonstrate an impairment of brain cholesterol metabolism in SCA3 and suggest that this dysfunction may be related with the observed reduction of CYP46A1 expression, in the context of the disease.

\section{CYP46A1 overexpression alleviates neuropathology in a lentiviral-based SCA3 mouse model}

Decreased expression of CYP46A1 in the brain of SCA3 patients and mice prompted us to evaluate whether restoring CYP46A1 expression and upregulating the cholesterol metabolism pathway could improve mutant ataxin-3 clearance, therefore, delaying the establishment of SCA3associated neuropathology. This possibility was initially addressed using a lentiviral (LV)-based mouse model of SCA3 [4, 48]. We co-overexpressed lentiviral vectors 

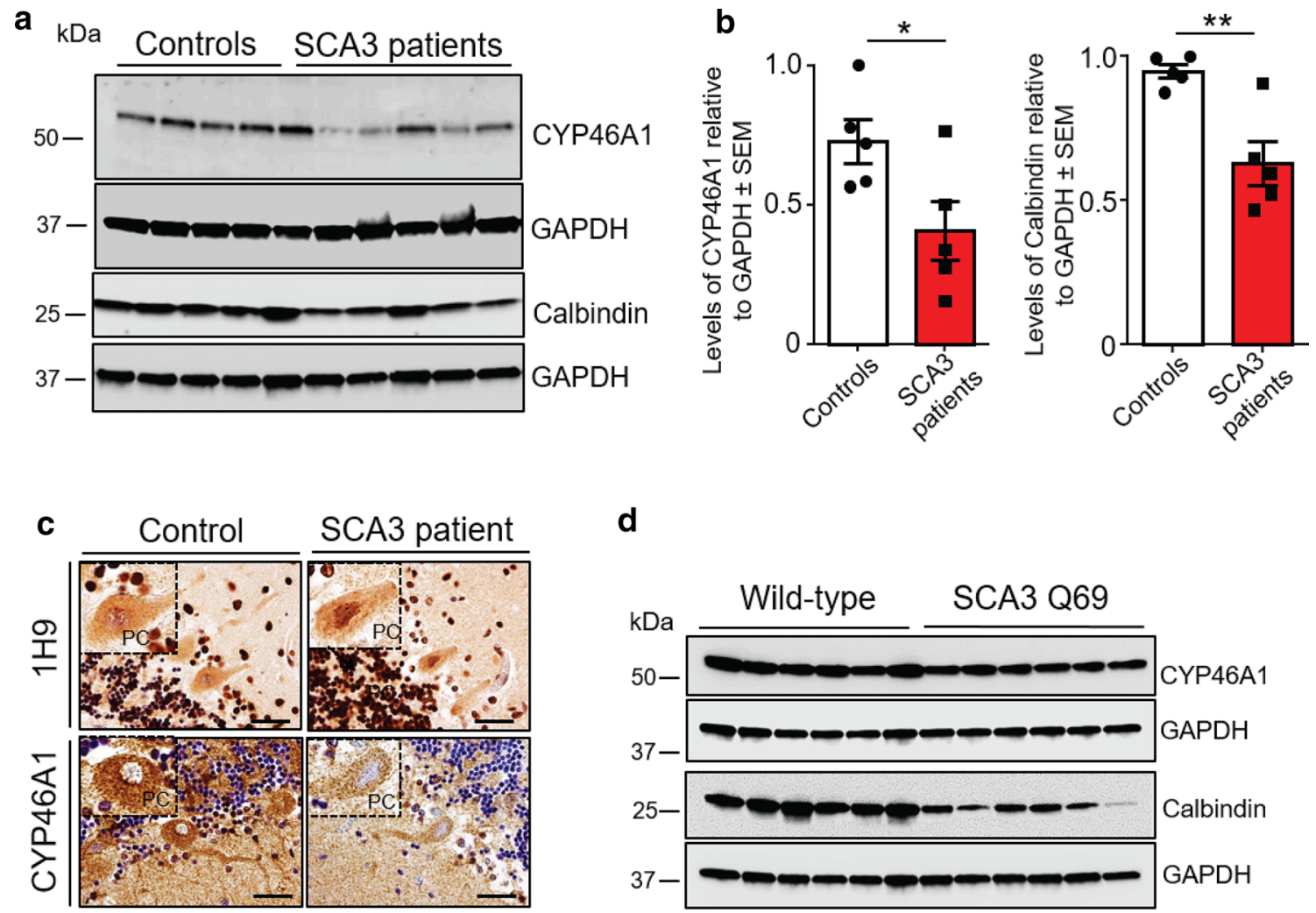

d
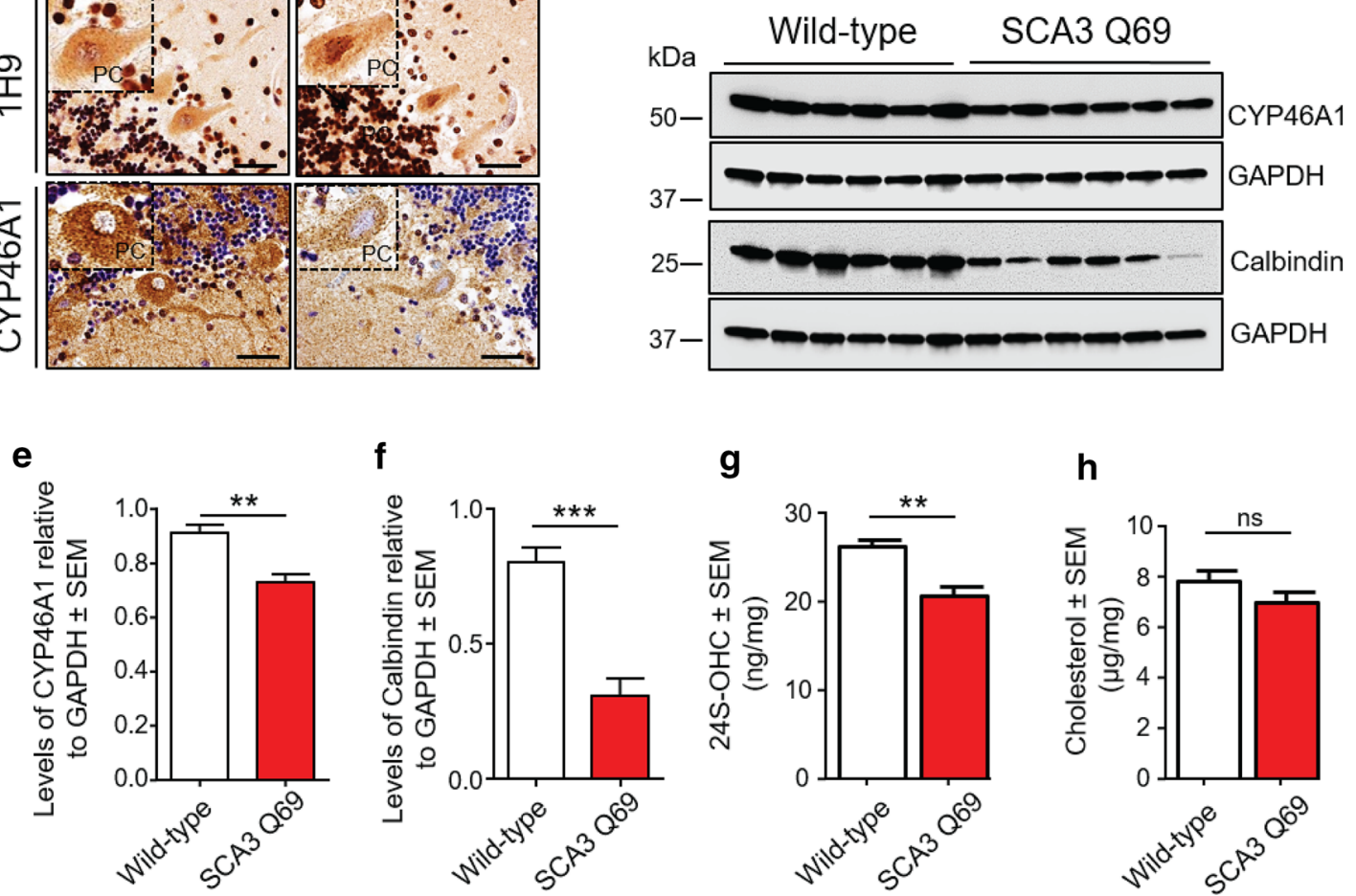

Fig. 1 CYP46A1 levels are decreased in the cerebellar cortex of SCA3 patients and SCA3 transgenic mice. a, b Representative western blot of samples from the cerebellar cortex of SCA3/MJD patients $(n=5)$ and healthy controls $(n=5)$ showing decreased levels of CYP46A1 (44\%) (Student's $t$ test; $P=0.04$ ). Levels of the Purkinje cell (PC) marker calbindin decreased $34 \%$ in SCA3 patients relatively to controls (Student's $t$ test). c Representative immunohistochemistry in human post-mortem brain slices with an anti-ataxin-3 antibody (1H9) showing ataxin-3 accumulation in the nucleus of a SCA3 PC, while remaining cytosolic in control PCs. CYP46A1 immunoreactivity is decreased in a SCA3 PC (Bars: $20 \mu \mathrm{m})$. d, e Western blot of

encoding human full-length mutant ataxin-3 (LV-based SCA3 mouse) and AAVs encoding CYP46A1 or control GFP in mice striatum (Fig. 2a, b) and sacrificed the animals 2 months post-injection. As expected, HA-directed immunohistochemistry revealed the presence of the HA tag in

whole cerebellar lysates from 3-month-old SCA3 Q69 mice $(n=6)$ and wild-type littermates $(n=6)$, showing a decrease in CYP46A1 levels ( 20\%; Student's $t$ test; $P=0.0013)$ in the cerebellum of SCA3 Q69 mice compared to control littermates. d, $\mathbf{f}$ The levels of the calbindin were decreased in SCA3 Q69 mice compared to controls. $\mathrm{g}$ Gas chromatography-mass spectrometry (GC/MS) in whole cerebellar extracts from SCA3 Q69 mice $(n=6)$ and controls $(n=6)$ showed a statistically significant decrease of $24 \mathrm{~S}-\mathrm{OHC}$ in SCA3 Q69 mice relatively to wild-type littermates (Student's $t$ test, $P=0.002$ ). h No statistically significant differences were observed for the cerebellar cholesterol content

AAVrh10-CYP46A1-injected LV-SCA3 mice but not in the control mice injected with AAVrh10-GFP (Fig. 2c). Conversely, control mice showed a robust and widespread GFP brain immunoreactivity, whereas no GFP-positive signal was detected in AAVrh10-CYP46A1-injected mice (Fig. 2d). In 
the LV-based SCA3 mouse model, immunohistochemistry (1H9 antibody) and quantitative analysis of mutant ataxin3 -positive inclusions revealed a statistically significant decrease (59\%) in the number of ataxin-3-positive inclusions present in animals expressing CYP46A1 (2751 \pm 267.8 ; $n=8$ ), compared to the control group (AAVrh10-GFP; $6684 \pm 717.2$ inclusions; $n=8$; Student's $t$ test, $P=0.0002$ ) (Fig. 2e, f, i). We also demonstrated a statistically significant decrease in the size of inclusions (47\%) in AAVrh10CYP46A1-treated LV-SCA3 mice $(59.29 \pm 4.67$ arbitrary units (a.u.); $n=8$ ) compared to LV-SCA3 mice injected with the control AAVrh10-GFP vector $(111.4 \pm 3.96$ a.u.; $n=8$; Student's $t$ test, $P<0.0001$ ) (Fig. $2 \mathrm{j}$ ). These results were complemented with an anti-ubiquitin immunostaining that demonstrated a reduction $(46 \%)$ in the number of ubiquitinated inclusions in LV-SCA3 mice injected with AAVrh10CYP46A1 (2008 \pm 185 inclusions; $n=8)$ relatively to control mice (3702 \pm 266 inclusions; $n=8$; Student's $t$ test, $P=0.0001$ ) (Fig. 2g, k).

In view of these results, we next investigated whether CYP46A1 overexpression would confer neuroprotection in the LV-SCA3 mouse model. LV-based mutant ataxin-3 overexpression in the mouse striatum promotes depletion of the neuronal marker DARPP-32 that can be immunohistochemically quantified [4]. AAVrh10-CYP46A1 overexpression led to a statistically significant reduction (48\%) of neuronal marker loss, as assessed by the decreased volume of DARPP-32-depleted region at 2 months $\left(0.48 \pm 0.77 \mathrm{~mm}^{3}\right.$; $n=8)$ relatively to controls $\left(0.91 \pm 0.14 \mathrm{~mm}^{3} ; n=8\right.$; Student's $t$ test; $P=0.015$ ) (Fig. 2h, 1). Laser confocal microscopy further demonstrated a reduction in inclusions size in the cell nucleus (DAPI staining; Fig. 2m). Overall, these data suggest that CYP46A1 overexpression promotes the clearance of aggregated human mutant ataxin-3 and elicits neuroprotection in a LV-SCA3 mouse model.

\section{AAV-mediated CYP46A1 gene therapy improves neuropathology and motor coordination in SCA3 transgenic mice}

Next, we aimed to investigate if CYP46A1 upregulation would alleviate motor and cerebellar deficits after the onset of the disease, using a well-established transgenic SCA3 mouse model [71]. This SCA3 Q69 transgenic mouse model is characterized by early disease sign onset and a severe motor and neuropathological phenotype, thus being particularly suitable for testing molecular therapies [42, 47, 49]. AAVrh10 encoding CYP46A1 or GFP were injected into the cerebella of adult 6-week-old mice (Suppl Fig. 2/Online Resource 4), at which age animals already display marked neuropathological abnormalities, namely a significant loss of PCs and the formation of HA-positive aggregates in these cells (Suppl Fig. 3; Online Resource 5), as well as motor impairments observable by the rotarod test, as compared to wild-type mice (WT) [53]. We observed that HA-CYP46A1 was expressed in mouse cerebellum already at 1 week postinjection and that the levels of the protein increased $\sim$ twofold by 4 weeks after the surgery (Suppl Fig. 4a/Online Resource 6). Moreover, 10 weeks after AAVrh10-CYP46A1 injection CYP46A1 was still being expressed, mainly in PCs (black arrows) and cerebellar neurons of the molecular layer (white arrows) (Suppl Fig. 4b/Online Resource 6 and Suppl Fig. 5/Online resource 7), and there is a clear tendency for increase of its levels in SCA3 Q69 mice injected with AAVrh10-CYP46A1, as compared with AAVrh10GFP-injected mice (Suppl Fig. 4c/Online Resource 6). Motor performance of injected SCA3-transgenic mice was evaluated every $2 / 4$ weeks, until 10 weeks after treatment (Fig. 3a-c). The rotarod test at constant speed (Fig. 3a) and the accelerated rotarod test (Fig. 3b) showed that, throughout the study, mice overexpressing CYP46A1 had a better motor performance as compared to GFP-injected mice, with significant differences observed at 10 weeks after surgery, for both rotarod tests (Fig. 3a, expressed by the latency to fall from the apparatus: AAVrh10-CYP46A1 $53.07 \mathrm{~s}$ versus AAVrh10-GFP $17.48 \mathrm{~s}$ for constant velocity). This improvement in motor functions was further demonstrated by the foot printing pattern test, namely by the footprint overlap and hind base test. Mice treated with AAVrh10-CYP46A1 showed a tendency for a better hind base performance at 10 weeks after AAVrh10-CYP46A1 injection (Fig. 3c, AAVrh10-CYP46A1: $3.17 \pm 0.07 \mathrm{~cm}$ versus AAVrh10-GFP: $3.40 \pm 0.11 \mathrm{~cm})$.

To investigate whether motor function improvements were accompanied by neuropathology amelioration, we next evaluated the number of aggregates, Purkinje cell number and volume of the cerebellar lobules of injected mice. In accordance with the behavioral data, we found that the average number of ubiquitin-positive aggregates was significantly reduced $(\sim 50 \%)$ in the entire cerebellar cortex and lobules IX and X from AAVrh10-CYP46A1-injected SCA3 Q69 mice, relatively to AAVrh10-GFP-injected SCA3 Q69 mice (Fig. 3d upper panel, e; Suppl Fig. 6/Online Resource $8)$. The SCA 3 transgenic mouse model used is characterized by loss of PCs and strong disorganization of the cerebellar cortex [53]. Importantly, the average number of PCs in the whole cerebellum of SCA3 Q69 animals injected with AAVrh10-CYP46A1 was significantly higher than that of controls $(1627 \pm 84.8$ versus $1213 \pm 125.9$, respectively) (Fig. 3d lower panel, f; Suppl Fig. 7a-f/Online Resource 9). Moreover, volume of cerebellar lobules IX and X (IX: $0.1024 \pm 0.007$ versus $0.1242 \pm 0.003 ; \mathrm{X}: 0.063 \pm 0.004$ versus $0.079 \pm 0.005$ ) (Fig. $3 \mathrm{~g}-\mathrm{i}$ ) and thickness of the granular layer was significantly larger in AAVrh10-CYP46A1injected mice, compared to AAVrh10-GFP SCA3 Q69 mice (Suppl Fig. 7 g-h/Online Resource 9). Altogether, our 
a

LV-MUTATXN3 LV-MUTATXN3

AAVrh10-CYP46A1 AAVrh10-GFP

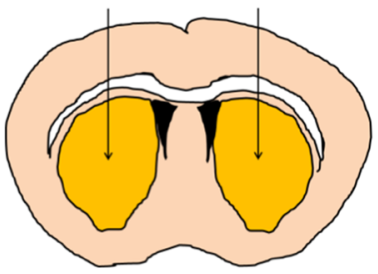

b
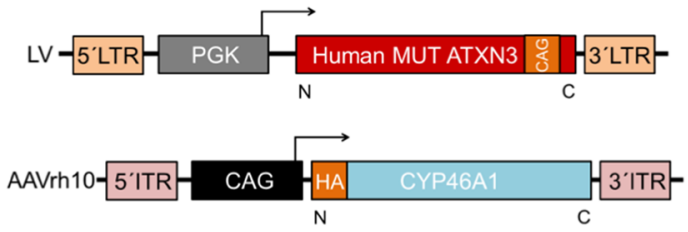

AAVrh10-5'ITR CAG GFP

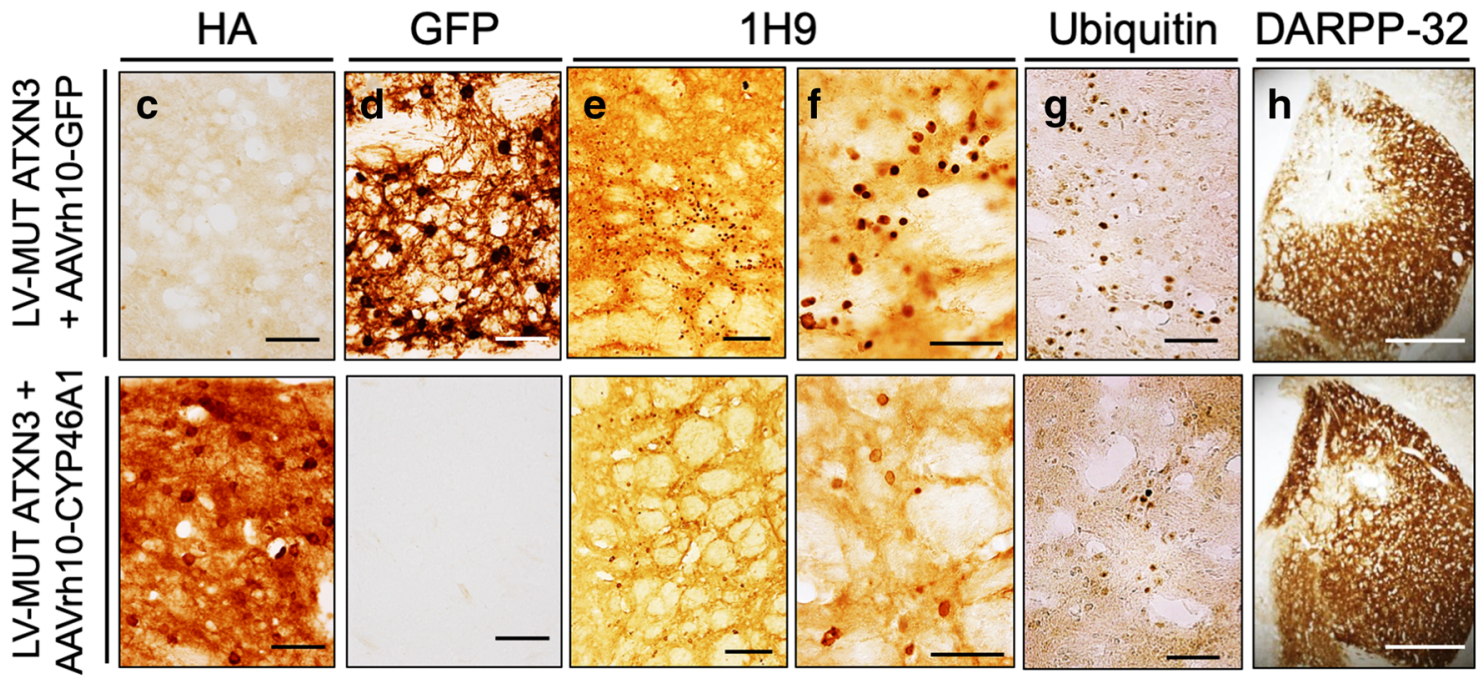

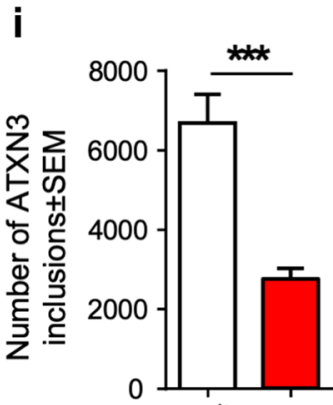

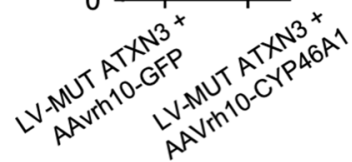

m

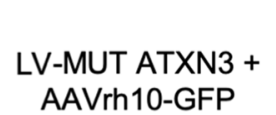

LV-MUT ATXN3 + AAVrh10-CYP46A1
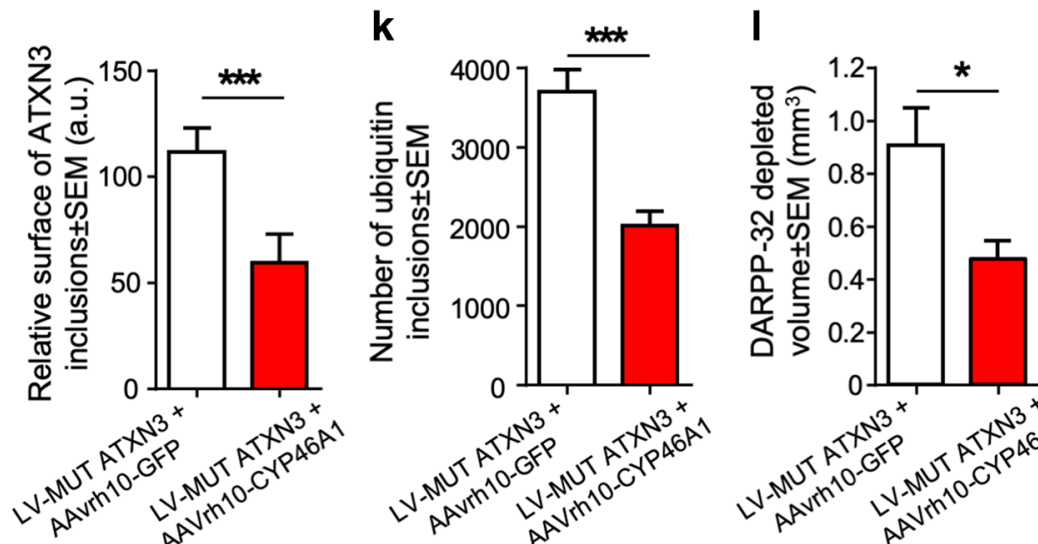

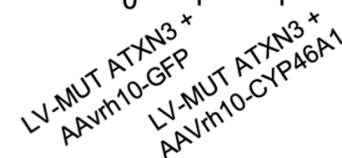

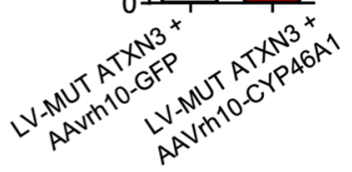
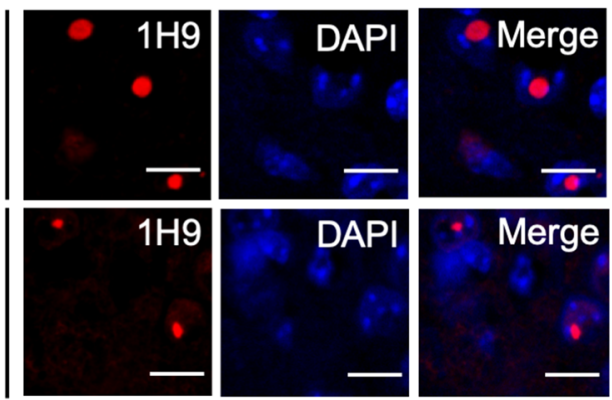
4Fig. 2 CYP46A1 clears mutant human ataxin-3 and confers neuroprotection in a lentiviral-based SCA3 mouse model. a, b Schematic representation of the mouse striatal co-injection procedure using lentiviral vectors encoding mutant ATXN3 (LV-MUT ATXN3) driven by the phosphoglycerate-1 (PGK-1) promoter along with AAVrh10CYP46A1 or control AAVrh10-GFP, whose expression is driven by the chicken $\beta$-actin promoter. c Immunohistochemistry of mouse striatal brain slices revealed the HA tag (HA-CYP46A1) in AAVrh10CYP46A1-injected LV-MJD mice and $\mathbf{d}$ the GFP immunostaining in AAVrh10-GFP-injected LV-MJD mice. e, f Immunohistochemistry with the anti-ataxin-3 (1H9) antibody revealed mutant ataxin-3 inclusions in LV-MUT ATXN3 mice co-injected with AAVrh10-GFP, whereas a reduction in mutant ataxin-3 deposition was observed in LV-MUT ATXN3 mice co-injected with AAVrh10-CYP46A1 (quantification of the number and relative surface of inclusions in $\mathbf{i}, \mathbf{j}$, respectively; $n=8$; Student's $t$ test); g, k AAVrh10-CYP46A1 reduces the number of ubiquitinated inclusions, as well as $(\mathbf{h}, \mathbf{l})$ the DARPP-32-depleted volume relatively to LV-MUT ATXN3 mice injected with control AAVrh10-GFP ( $n=8$; Student's $t$ test). m Laser confocal microscopy showing nuclear deposition of mutant ataxin-3 aggregates (red) in striatal cells of mice co-injected with LV-MUT ATXN3 and AAVrh10-GFP, relatively to LV-MUT ATXN3 mice coinjected with AAVrh10-CYP46A1. Nuclei were counterstained with 4',6-diamidino-2-phenylindole (DAPI). Bars: c, d $50 \mu \mathrm{m}$; e $100 \mu \mathrm{m}, \mathbf{f}$, g $50 \mu \mathrm{m}, \mathbf{h} 1 \mathrm{~mm}, \mathbf{l} 20 \mu \mathrm{m}$

results show that the upregulation of CYP46A1 in a severe SCA3 transgenic mouse model mediates a robust alleviation of the characteristic motor phenotype and neuropathological abnormalities.

\section{In vivo inhibition of CYP46A1 perturbs brain cholesterol metabolism and induces a SCA3-like phenotype in normal mice}

To get further insight into the role of cholesterol metabolism dysfunction and of CYP46A1 expression in SCA3, we evaluated whether CYP46A1 downregulation in vivo resulted in an SCA3 similar phenotype, in WT mice. Since the striatum is affected in SCA3 $[4,31,55]$ we analyzed the impact of CYP46A1 silencing in this brain region, in C57BL6J mice locally injected with AAV5-shCYP46A1 $(n=11)$ or control AAV5-shScramble $(n=11)$ (Fig. 4a). We first confirmed that AAV5-shCYP46A1 delivery induced a reduction of CYP46A1 expression $(\sim 60 \%)$ in the striatum of injected animals (Fig. 4b, c) compared to controls. Importantly, the decreased expression of CYP46A1 was associated with modifications of the lipidomic profile. Through GC-MS analysis of striatal samples, we observed a $41 \%$ decrease in the levels of $24 \mathrm{~S}-\mathrm{OHC}$ in the striatum of AAV-shCYP46A1-injected mice (Student's $t$ test; $P=0.0037$ ) relatively to AAV-shScramble-injected mice (Fig. 4d), while no differences were detected for cholesterol levels (Fig. 4e). Similarly, the levels of lanosterol remained unchanged (Suppl Fig. 8/Online Resource 10). Of note, precursors from both Kandutsch-Russell and Bloch pathways were decreased. A trend for decreased lathosterol, a significant decrease of 7-DHC (39\%) (Student's $t$ test; $P<0.0001)$ and 8-DHC (38.5\%) (Student's $t$ test; $P=0.0017$ ) from the Kandutsch-Russell pathway and a decrease ( $25 \%$; Student's $t$ test; $P=0.014)$ of desmosterol, a cholesterol intermediate of the Bloch pathway, were observed in AAV-shCYP46A1-injected mice (Suppl Fig. 8/Online Resource 10).

Motor and balance performances were analyzed using the rotarod test. One-month post-injection, no statistically significant differences were observed between groups; however, 3-months post-injection a statistically significant decrease in latency to fall (Fig. 4f; $P=0.035$; twoway ANOVA) was observed in AAV-shCYP46A1-injected mice relatively to AAV-shScramble-injected controls. Animals were also tested in the open field task test 3 months post-injection. Distance traveled and speed did not differ between groups (Suppl Fig. 9a/Online Resource 11), but AAV-shCYP46A1-injected mice displayed a trend towards a decreased ratio of the time spent in center/periphery (ratio C/P; Student's $t$ test, $P=0.10$; Suppl Fig. 9a/Online Resource 11), suggesting reduced anxiety. The elevated plus maze test revealed that, for the same traveled distance, both the time spent (Student's $t$ test; $P=0.0054$ ) and the frequency of entries (Student's $t$ test; $P=0.0098$; Suppl Fig. 9b/Online Resource 11) in the open arms were increased in AAV-shCYP46A1 mice $(n=8)$ relatively to control AAV-shScramble littermates $(n=8)$, also suggesting reduced anxiety.

Concerning the neuropathological profile of injected mice, fluorescence microscopy revealed strong depletion of NeuN-positive immunoreactivity in mouse striata transduced with AAV-shCYP46A1, whereas no cell loss was observed in AAV-shScramble-transduced mice (Fig. 4g). Moreover, AAV-shCYP46A1 expression induced strong striatal astrocytic (GFAP marker) and microglial (Iba-1 and CD68 markers) activation and inducible nitric oxide synthase (iNOS) enhancement relatively to control mice (Fig. 4h, i; Suppl Fig. 10a/Online Resource 12). We also observed an increased number of caspase-3-positive cells (Fig. 4j) and pycnotic nuclei, visible on hematoxylin-eosin stained sections (Fig. 4k), coalescence of the striatal capsule (Brightfield; Fig. 4l) and increased levels of nitrotyrosine (Suppl Fig. 10b/Online Resource 12), suggesting cell injury and striatal degeneration after striatal delivery of AAV-shCYP46A1, whereas no such cell degeneration was observed on AAV-shScramble-injected mice striatal sections.

Taken together, the observed effects of CYP46A1 downregulation in mouse striatum suggest that reduced CYP46A1 activity leads to severe neuropathological abnormalities and clinical consequences resembling what has been described for SCA3. 
a

Rotarod (constant speed)

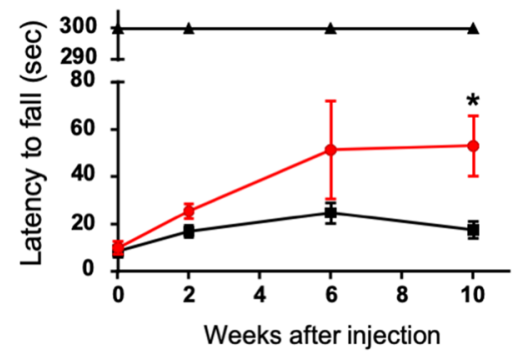

$+W T \quad \rightarrow \quad$ SCA3 Q69 AAVrh10-CYP46A1

d
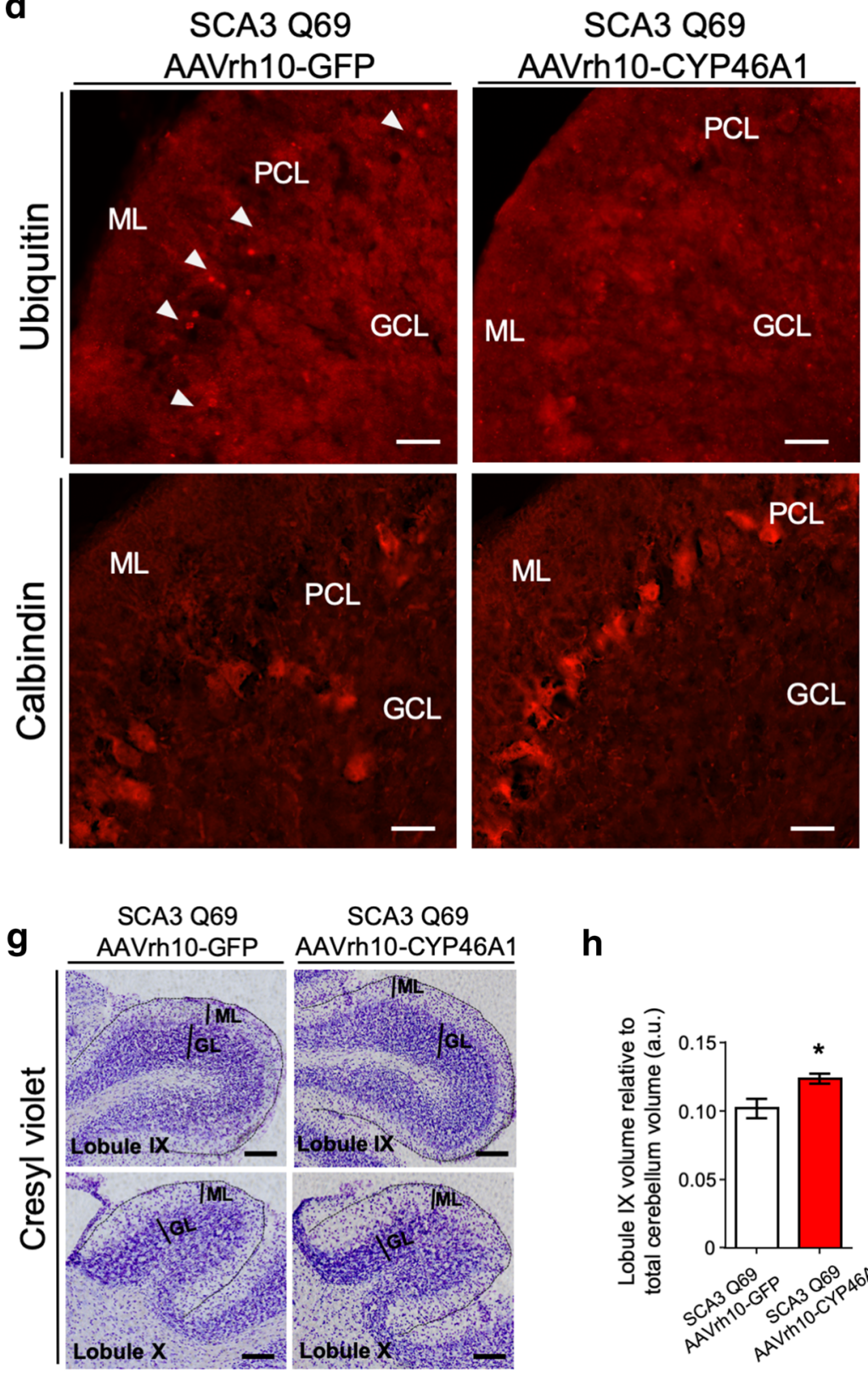

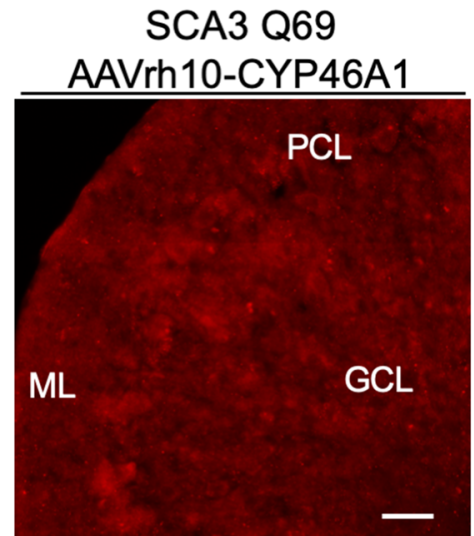

h

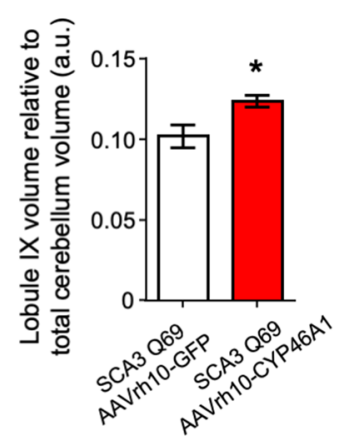

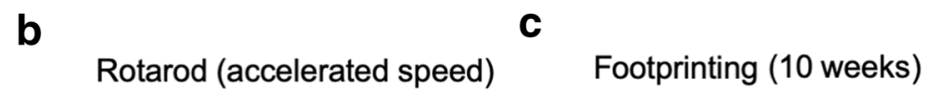
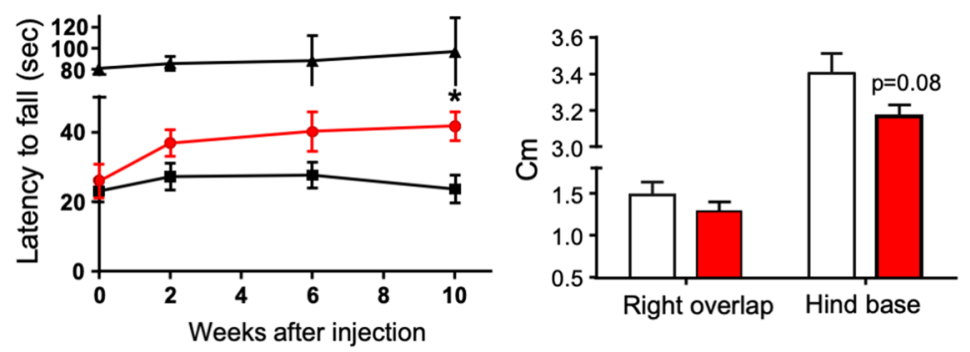

- SCA3 Q69

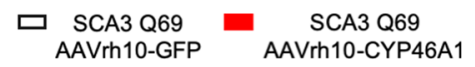

e

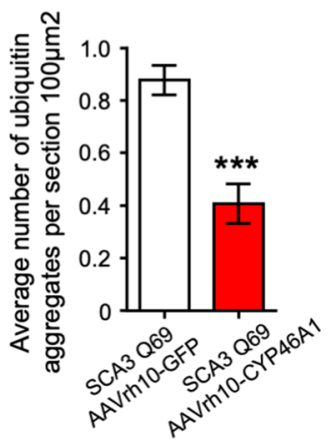

f
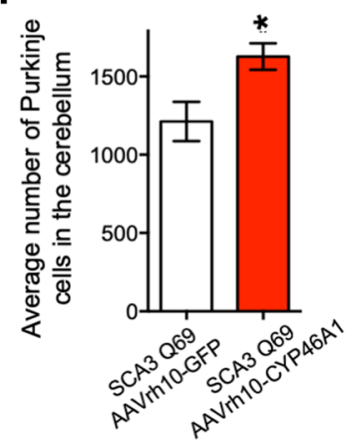

i

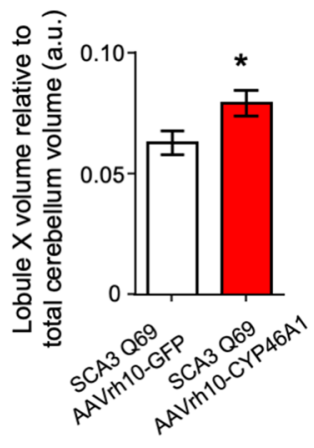


4Fig. 3 CYP46A1 overexpression improves motor coordination and neuropathology impairments in SCA3 Q69 transgenic mice. Noninjected wild-type mice (WT; $n=4$ ) and SCA3 Q69 transgenic mice injected with AAVrh10 encoding for CYP46A1 (AAVrh10CYP46A1; $n=10$ ) or GFP (AAVrh10-GFP; $n=9$ ) were assessed for motor coordination performance over 10 weeks. Mice were tested for a rotarod at constant velocity, $\mathbf{b}$ accelerated rotarod and $\mathbf{c}$ footprint overlap and hind base measures (footprinting test). A significant improvement of motor performance in the transgenic SCA3 Q69 mice treated with AAVrh10-CYP46A1 as compared to the control mice (AAVrh10-GFP) was observed. d At 10 weeks post-injection, mice were killed and the brain was immunohistochemically labeled for neuropathological markers. Representative fluorescence microscopy images of ubiquitin staining for AAVrh10-CYP46A1- and AAVrh10GFP-injected mice (upper panel), and for calbindin staining depicting a detail of lobule X (lower panel) are presented. e CYP46A1 overexpression led to a significant decrease in the average number of ubiquitin aggregates per section in the entire cerebellum, compared to control animals (AAVrh10-CYP46A1/AAVrh10-GFP; $n=6$ ). f The average number of Purkinje cells in the entire cerebellum was significantly higher in animals injected with AAVrh10-CYP46A1 $(n=6)$, compared to AAVrh10-GFP-injected mice $(n=6)$. g Representative microscopy images of cresyl violet staining depicting cerebellar lobules IX and X in both groups of injected mice. $\mathbf{h}, \mathbf{i}$ The cerebellar volume was significantly larger in lobules IX and $\mathrm{X}$ of AAVrh10-CYP46A1-injected $(n=6)$, compared to mice injected with AAVrh10-GFP $(n=6)$. Data are presented as mean \pm SEM. $* p<0.05$; $* * p<0.01 ; * * * p<0.001$; two-way ANOVA analysis with Bonferroni post-test or unpaired Student's $t$ test. Bars: d $20 \mu \mathrm{m}$; j $50 \mu \mathrm{m}$. PCL Purkinje cells, $G C L$ granular layer, $M L$ molecular layer

\section{Silencing CYP46A1 in the mouse brain impairs the endosomal-lysosomal pathway}

There is evidence that the endosomal/lysosomal pathway is impaired in polyQ-SCAs, in particular in SCA3 [64] and SCA7 [1]. Furthermore, we have previously shown that silencing CYP46A1 in the mouse hippocampus induces perturbations in endosomal trafficking leading to the formation of enlarged lysosomes [24]. In addition, it has been reported that late endosomal cholesterol accumulation leads to impaired intra-endosomal trafficking [65]. To investigate whether such endosomal trafficking impairments also occurred in the AAV5-shCYP46A1-injected mouse striatum, we performed immunostaining for endosomal and lysosomal markers, in particular for early endosomes (Rab5), late endosomes (Rab7) and lysosomes (lysosomal-associated membrane protein 1; LAMP-1) (Fig. 5a). An increase of endosome and lysosome immunostaining was observed in the striatum of mice receiving AAV5-shCYP46A1, relatively to controls, where these organelles were hardly detected (Fig. 5a). In addition, co-localization between GFP and cathepsin-D (Cath-D), the main aspartic protease present in lysosomes, had a fine granular morphology in AAV5-shScramble-transduced neurons, whereas it was more intense in striatal cells expressing AAV5-shCYP46A1 (Fig. 5b). Ubiquitin deposits were observed in the cytoplasm of neurons transduced with AAV5-shCYP46A1, while standard widespread cytosolic expression of ubiquitin was present in neurons from areas transduced with control AAV5-shScramble (Fig. 5c), suggesting impairment in protein quality-control pathways. Moreover, ultrastructural analysis using electron microscopy revealed that AAVshCYP46A1-transduced neurons exhibited non-standard, enlarged lysosomes and autophagolysosomes enclosing dark content containing abundant electron-dense vesicles and mitochondria in the lumen (Fig. 5d). On the contrary, primary lysosomes of AAV-shScramble-transduced neurons had standard morphology, comprising homogeneous and fine granular content (Fig. 5d).

\section{CYP46A1 induces autophagy in vitro and in the mouse brain and clears mutant ataxin-3 aggregates}

These data showing that neuronal CYP46A1 inhibition impaired endosomal-lysosomal membrane trafficking, which is abnormal in several SCAs, prompted us to evaluate whether the mechanism through which CYP46A1 alleviated the SCA3 phenotype involved activation of the autophagic pathway. For this, we used Neuro2A cells expressing mutant ataxin-3 (EGFP-Q84) to analyze the impact of CYP46A1 expression on expanded ataxin-3 aggregates and on autophagy markers. Western blot analysis of the levels of mutant ataxin-3 species revealed that CYP46A1 significantly reduced both aggregated $(1 \pm 0.0$ versus $0.449 \pm 0.11$; $n=4$; Fig. $6 \mathrm{a}-\mathrm{c})$ and soluble mutant ataxin-3 $(1 \pm 0.0$ versus $0.09 \pm 0.06 ; n=4$; Fig. $6 c$ ), whereas mouse endogenous ataxin-3 levels remained unaltered (Suppl Fig. 11a, b/Online Resource 13). Moreover, the number of cells with mutant ataxin-3 aggregates was significantly reduced upon CYP46A1 overexpression (Fig. 6d, e). Expression of CYP46A1 did not produce any alteration in cell viability, comparing to non-transfected cells; similarly, no significant improvement in cell viability was observed when CYP46A1 was co-expressed with mutant ataxin-3, compared to the control condition (Suppl Fig. 12/Online Resource 14). Interestingly, CYP46A1 was also able to significantly reduce the levels of SCA2-causing protein mutant ataxin-2 in transfected cells, (Suppl Fig. 11c, d; Online Resource 13) and decreased the number of cells with mutant ataxin-2 aggregates (Suppl Fig. 11e, f/Online Resource 13).

We also found that CYP46A1 overexpression in Neuro2A cells expressing mutant ataxin-3 significantly increased LC3B-II levels $(2.12 \pm 0.35 ; n=4)$, compared to the control condition $(1 \pm 0.0 ; n=4)$. The increase in LC3B-II levels was even higher upon CYP46A1 overexpression than in the starvation condition (autophagy-positive control; $1.58 \pm 0.02 ; n=4$ ) (Fig. $6 \mathrm{~g}, \mathrm{~h}$ ). Importantly, in the presence of chloroquine (ChQ), which inhibits autophagic flux by decreasing the fusion of autophagosomes with lysosomes, 


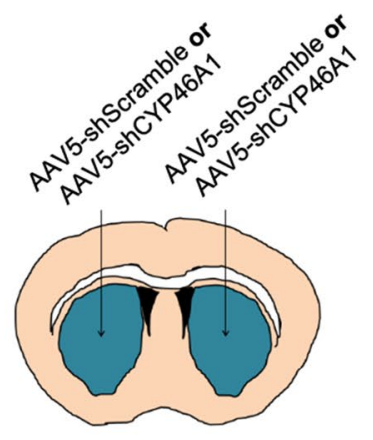

b

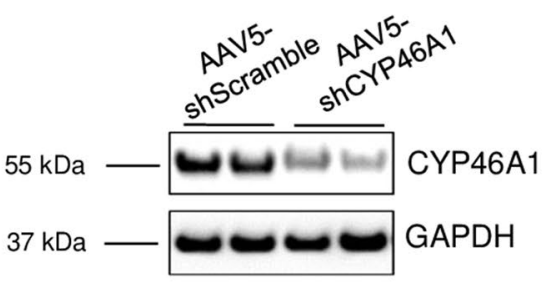

C

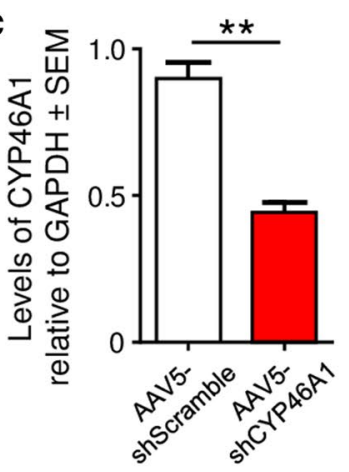

d

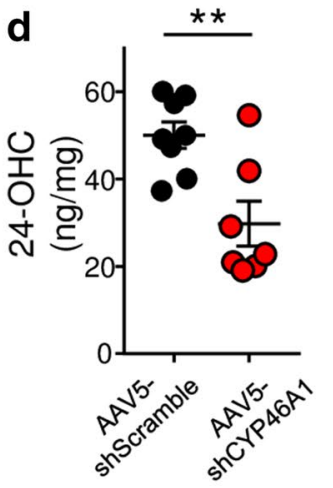

e

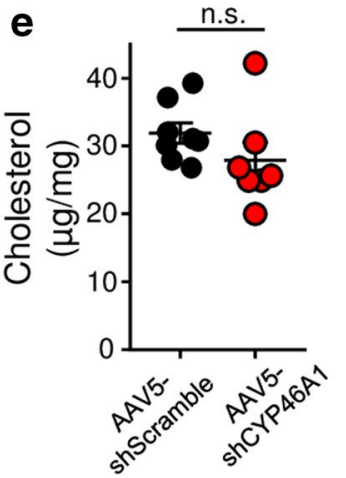

f

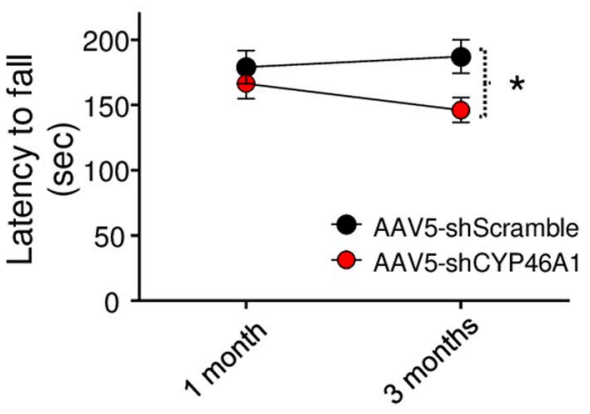

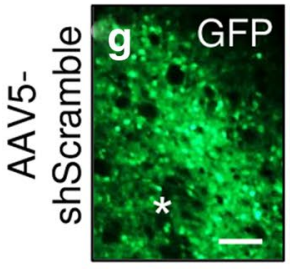
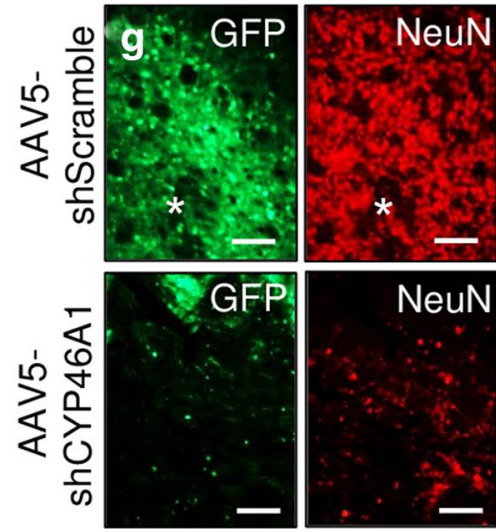
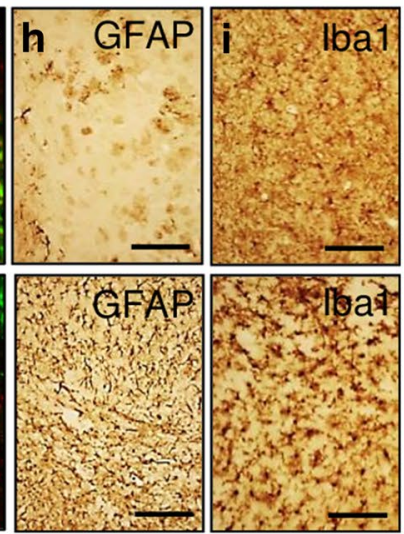
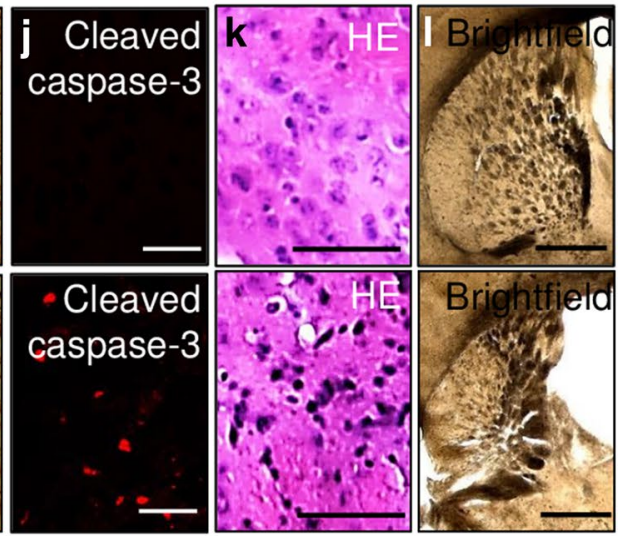

Fig. 4 Silencing CYP46A1 impairs brain cholesterol metabolism and induces motor impairment accompanied by striatal pathology, in wild-type mice. a Schematic representation of the mouse bilateral striatal injection of AAV5-shCYP46A1 or AAV5-shScramble, both vectors containing the GFP reporter gene. b, $\mathbf{c}$ Representative western blot showing a $\sim 60 \%$ of decrease in CYP46A1 levels in the striatum of mice injected with AAV5-shCYP46A1 ( $n=3 /$ group). d Gas chromatography-mass spectrometry (GC-MS) analysis showing a statistically significant decrease in the levels of $24 \mathrm{~S}-\mathrm{OHC}$ in the striatum of AAV-shCYP46A1-injected mice $(n=8)$ (Student's $t$ test; $P<0.0001)$, compared to AAV-shScramble mice $(n=8)$, e while levels of cholesterol remained unchanged. f Injection of AAV5-shCYP46A1 in the mouse striatum impaired motor behavior; rotarod assessment:

results followed the same trend, i.e., there was a significant increase in LC3B-II levels upon CYP46A1 overexpression $(6.74 \pm 1.31 ; n=4)$ compared to control condition $(2.83 \pm 0.44 ; n=4)$ (Fig. 6f, g). In addition, CYP46A1 no statistically significant differences between groups were observed at 1 month post-injection; at 3 months post-injection, shCYP46A1injected mice displayed a statistically significant decrease in latency to fall $(P=0.035$; Two-way ANOVA) relatively to controls. g Fluorescence microscopy showing decrease in striatal NeuN (red) immunostaining in AAV-shCYP46A1-injected mice, while NeuN (red) was preserved in AAV-shScramble mice (*needle track). $\mathbf{h}$ Increased activation of astrocytes (anti-GFAP immunostaining), i microglia (antiIba1 immunostaining), $\mathbf{j}$ increased number of caspase-3-positive cells and $\mathbf{k}$ pycnotic nuclei (Hematoxylin/Eosin-HE-coloration), $\mathbf{l}$ accompanied by coalescence of the striatum (Brightfield,), were observed in AAV-shCYP46A1-injected mice. Bars: $\mathbf{g}, \mathbf{h}$ and i: $20 \mu \mathrm{m} ; \mathbf{j} 20 \mu \mathrm{m}$; k $50 \mu \mathrm{m} ; 11 \mathrm{~mm}$

overexpression significantly increased the autophagic flux, highlighted by an increase in the LC3B-II net flux ( $2.80 \pm 0.16$ versus $1 \pm 0.0$ in the control condition; $n=4)$ (Fig. 6f, h). The levels of SQSTM1/p62, another important 

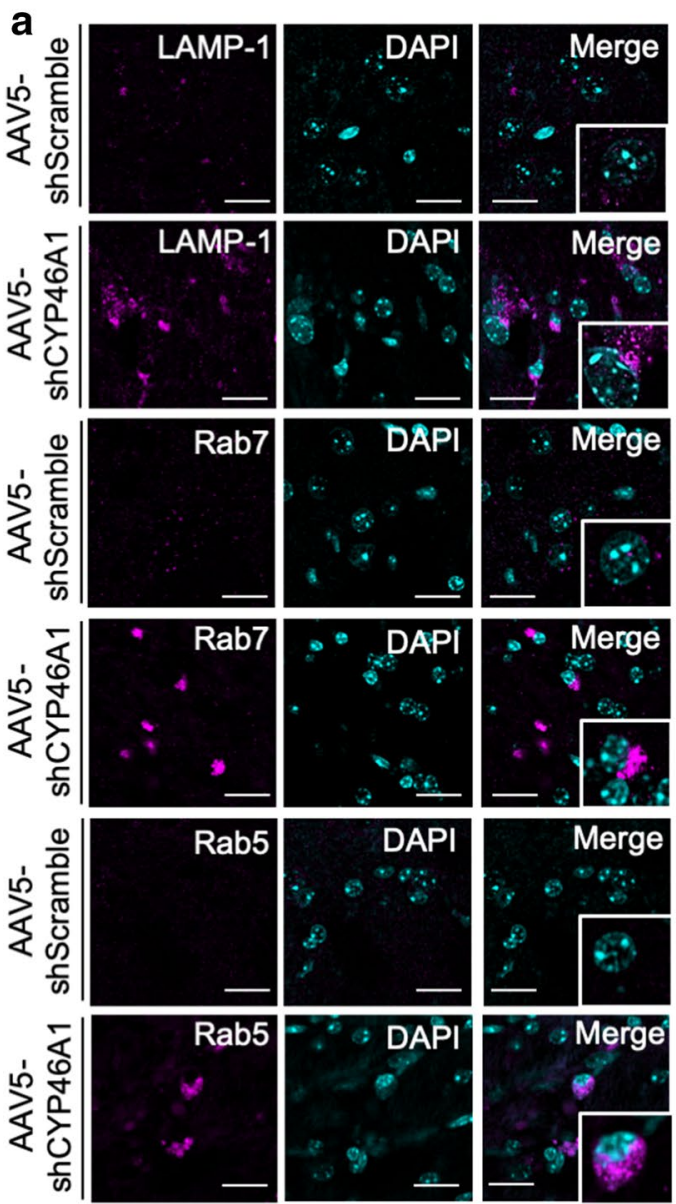

b
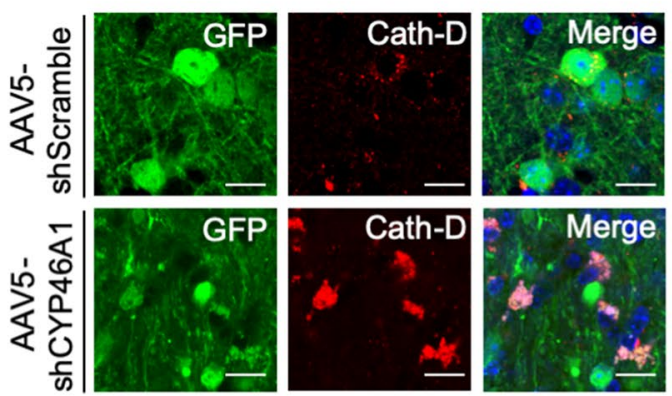

C
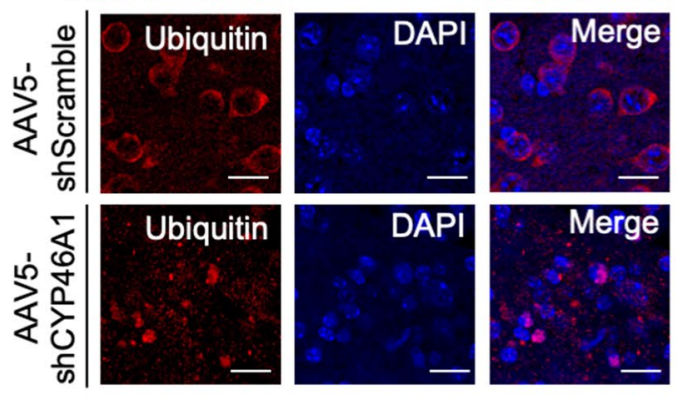

d

AAV5-shScramble

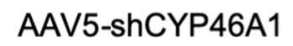

AAV5-shCYP46A1
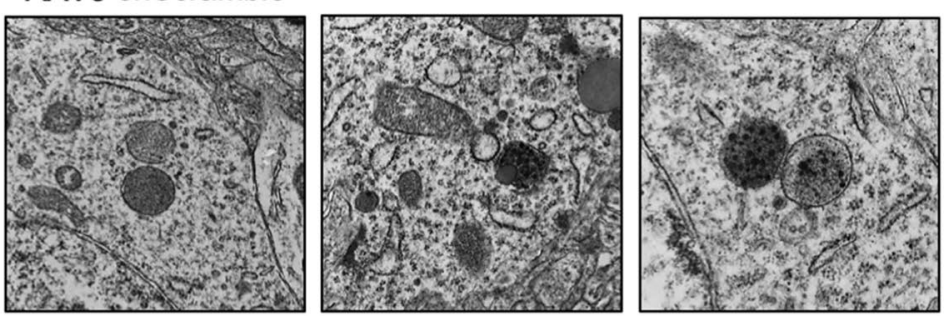

Fig. 5 Silencing CYP46A1 in the mouse brain promotes lysosome and endosomal accumulation. a Laser confocal microscopy showing robust accumulation of early endosomes (anti-Rab5; in violet), late endosomes (anti-Rab7; in violet) and lysosomes (lysosomalassociated membrane protein 1; LAMP-1; in violet), in striatal brain slices from C57/B16J mice injected with AAV5-GFP-shCYP46A1, relatively to $\mathrm{C} 57 / \mathrm{B} 16 \mathrm{~J}$ mice injected with control AAV5-GFPshScramble; $n=5$ /group. b Increased cathepsin-D (Cath-D; in red) immunostaining in striatal cells of mice receiving AAV5-GFPshCYP46A1 contrasting to a fine granular morphology in AAV5GFP-shScramble-positive striatal neurons from mice receiving AAV5-GFP-shCYP46A1; $n=5$ /group. c Anti-ubiquitin immunostain- ing showing ubiquitinated deposits (in red) in the cytosol of neurons transduced with AAV5-GFP-shCYP46A1, while standard cytoplasmic immunoreactivity (in red) was detected in AAV5-GFP-shScramble cells. Nuclei were counterstained with DAPI; $n=5 /$ group. d Electron micrographs revealing non-standard enlarged lysosomes and autophagolysosomes enclosing dark content containing abundant electron-dense vesicles and mitochondria in the lumen of neurons transduced with AAV-GFP-shCYP46A1, relatively to primary lysosomes with homogeneous morphology and fine granular content observed in AAV-GFP-shScramble neurons; $n=3$ /group. Bars: $20 \mu \mathrm{M}(\mathbf{a}-\mathbf{c})$

autolysosomes upon CYP46A1 expression compared to the control condition (Suppl Fig. 13b, c/Online Resource 15), being even more pronounced than in the starvation positive control condition. Altogether, both data from western-blot and immunocytochemistry assays strongly support the activation of autophagy upon the expression of CYP46A1.

To further assess the specificity of CYP46A1 in activating autophagy we performed a similar experiment, but inhibiting the ubiquitin-proteasome system (UPS) using MG132 and leading to the accumulation of polyubiquitinated proteins (Suppl Fig. 14/Online Resource 16). The results show that CYP46A1 reduced the levels of mutant ataxin-3 (both of 

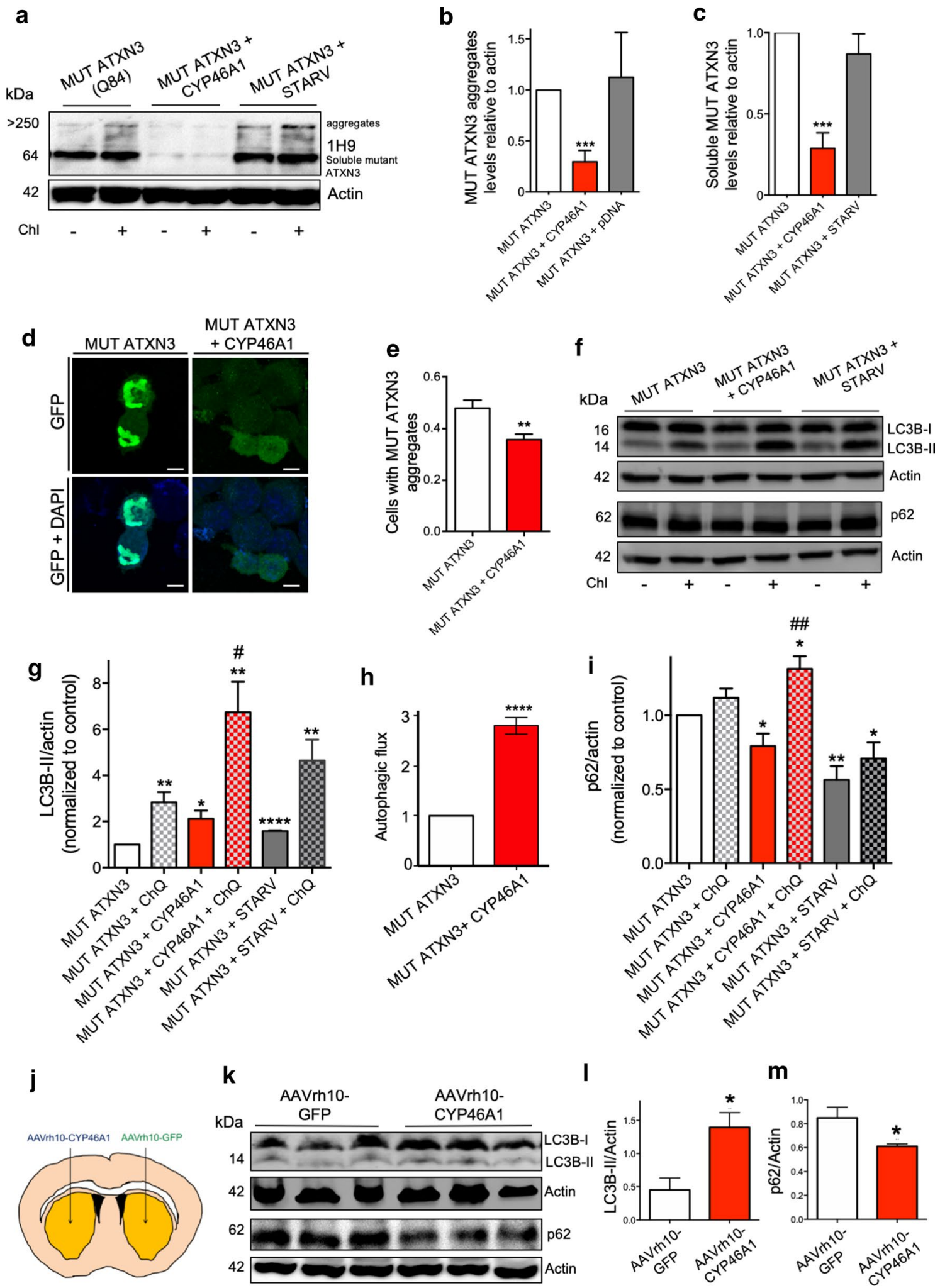

aggregates and of soluble species) regardless of UPS inhibition (Suppl Fig. 14a-c/Online Resource 16). In accordance with these results, the number of mutant ataxin-3 aggregates counted in immunofluorescence assays was decreased upon
CYP46A1 overexpression with or without UPS inhibition (Suppl Fig. 14d/Online Resource 16). These results clearly highlight that CYP46A1 action is through autophagy activation, independent of UPS. 
4Fig. 6 CYP46A1 induces autophagy in vitro and in the mouse brain and clears mutant ataxin-3 in a Neuro2A cellular model of SCA3. a Representative western blot of protein lysates from Neuro2A cells expressing mutant ataxin-3 (MUT ATXN3; Q84) as control condition, expressing mutant ataxin-3 and CYP46A1 and from starvation condition as positive autophagy control. Membranes were probed with the anti-ataxin-3 (1H9) antibody. Optical densitometry analysis revealed that CYP46A1 overexpression decreased both $\mathbf{b}$ aggregated and c soluble species of mutant ataxin-3 ( $n=4$ independent experiments). d Representative confocal microscopy images of Neuro2A cells depicting mutant ataxin-3 aggregates in a control condition, whereas in cells treated with CYP46A1 no aggregates are observed. e The number of transfected cells with mutant ataxin-3 aggregates was significantly reduced upon CYP46A1 overexpression $(n=7$ independent experiments). f Representative western blot probed for LC3B and SQSTM1/p62 of protein lysates from Neuro2A cells expressing mutant ataxin-3 as control condition, expressing mutant ataxin-3 and CYP46A1 and from a starvation condition as positive autophagy control. g Optical densitometry analysis revealed that CYP46A1 increased the levels of LC3B-II, while its accumulation was detected in the presence of chloroquine (Chl). $\mathbf{h}$ The autophagy flux was robustly increased with CYP46A1 overexpression. i Optical densitometry analysis revealed that CYP46A1 overexpression significantly decreased the levels of SQSTM1/p62. j Schematic representation of the mouse striatal co-injection of AAVrh10-CYP46A1 and control AAVrh10-GFP. k Representative western blot probing for LC3B and SQSTM1/p62 in protein lysates obtained from the striatal punches of the injected mice $(n=4)$. I Optical densitometry analysis revealed that CYP46A1 increased the levels of LC3B-II in the mouse striatum, compared to the GFP-injected hemispheres. $\mathbf{m}$ Optical densitometry analysis revealed that CYP46A1 overexpression significantly decreased the levels of SQSTM1/p62 in vivo. Values are expressed as mean \pm SEM. $* P<0.05$, $* * P<0.01$; $* * * * P<0.0001$ relative to control condition; ${ }^{\#} P<0.05,{ }^{\# \#} P<0.01$ relative to MUT ATXN3 + CYP46A1 condition (Unpaired Student's $t$ test). Bars: d $5 \mu \mathrm{m}$

Altogether, our results show that CYP46A1 modulates the levels of two polyQ proteins: mutant ataxin-3 and mutant ataxin-2, reducing their levels through the specific activation of the autophagy pathway. Finally, to further explore the CYP46A1 ability to activate autophagy in vivo, we injected animals with AAVrh10-CYP46A1 in the striatum of one brain hemisphere, and in the contralateral hemisphere AAVrh10-GFP was administered as an internal control (Fig. 6j). Three weeks after injection the animals were sacrificed and brain striatal punches were analyzed by western blot to assess both LC3B and SQSTM1/p62 protein levels (Fig. 6k). Expression of CYP46A1 in the striatum led to a significant increase in the levels of LC3B-II $(1.39 \pm 0.22 ; n=4)$ and to a reduction in SQSTM1/p62 levels $(0.61 \pm 0.02 ; n=4)$, compared to the GFP-injected hemisphere $(0.45 \pm 0.18$ and $0.84 \pm 0.08$, respectively) (Fig. 61, m). These results show that CYP46A1 expression is able to activate the autophagy pathway in vivo.

\section{Discussion}

While cholesterol metabolism impairment has been widely associated with neurodegenerative disorders, the mechanisms through which altered cholesterol homeostasis affects neurodegeneration are not completely understood [72]. Cholesterol plays a pivotal role in brain function. The brain is the most cholesterol-enriched structure of a mammalian organism, containing roughly tenfold more cholesterol compared to any other organ [9]. A major part is contained in myelin sheets $(70 \%)$, where cholesterol is involved in the development of axons and dendrites, in synaptogenesis and in neuronal repair [17, 38, 40]. Besides the myelin compartment, cholesterol is a critical structural and regulatory element of brain cells and membranes. It is implicated in signal transduction, neurotransmitter release and membrane trafficking [8].

The abundance of cholesterol in the central nervous system mainly depends on its local synthesis and efflux rate and the metabolism of brain cholesterol is tightly regulated [8]. Cholesterol cannot permeate the BBB; therefore, it relies on CYP46A1 enzyme, which transforms cholesterol into 24-OHC, which can freely cross the BBB, allowing for the vast majority of brain cholesterol efflux [58]. CYP46A1 is a brain-specific enzyme highly expressed in neurons, in particular in the pyramidal neurons of the hippocampus and cortex, in the soma and dendritic trees of PCs of the cerebellum, in hippocampal and cerebellar interneurons, in particular in Golgi neurons present in the cerebellar granular cell layer as well as in neuropil [54]. CYP46A1 is a key neuronal enzyme in the brain and its role is emerging as a major contributor to the regulation of neuronal cholesterol metabolism [58]. Not only does CYP46A1 produce 24-OHC that can be extracted from the brain, but also in turn it activates the whole mevalonate pathway of cholesterol synthesis, in which the intermediate compounds are important contributors to many key cellular pathways, in particular endocytosis, autophagy and synaptic transmission [44]. Importantly, oxidative stress is a potent inductor of CYP46A1 expression [50] and CYP46A1 was shown to be a major stress response element in neurons [66]. Furthermore, it has been suggested that neurons that are exposed to a sudden rise in excitatory neurotransmission activate cholesterol efflux mechanisms to avoid cell death [67].

We previously showed that CYP46A1 levels are decreased in a mouse model of AD [16] and in affected brain regions of HD patients, as a result of severe dysregulated transcriptional activity [13]. This defect was associated with impairment of the cholesterol pathway and decreased 24-OHC production. Interestingly, transgenic KO mice for CYP46A1 show cognitive dysfunction 
[33] and CYP46A1 inhibition in the hippocampus of mice leads to hippocampal atrophy with amyloid and hyperphosphorylated Tau accumulation [24]. We thus hypothesized that levels of CYP46A1 could also be decreased in SCA3 and could account for some of the severe hallmarks of the pathology. We found that CYP46A1 levels are decreased in cerebellar samples from SCA3 patients and SCA 3 mice. Decreased CYP46A1 expression is associated with impaired brain cholesterol metabolism in SCA3 mice. Dysregulation of transcriptional activity is a common hallmark of SCAs; reviewed in [39]. Our results are in accordance with recent transcriptional data from MJD84.2 transgenic SCA3 mice, showing that cholesterol biosynthesis pathway is significantly altered in SCA3 [70].

Remarkably, reduction in CYP46A1 levels is accompanied by a 24-OHC decrease in the cerebellum of SCA3 mice, while total cerebellar cholesterol levels remain unchanged. These results confirm previous observations in Cyp46al knockout mice. In these animals, cholesterol synthesis is reduced by $40 \%$, while cholesterol levels are unchanged relatively to the levels found in wild-type mice, suggesting that in the absence of CYP46A1 enzyme, homeostasis is maintained by reducing the synthesis of new cholesterol [36]. These observations were also confirmed in AAVshCYP46A1-injected mice. CYP46A1 inhibition decreases 24-OHC levels and disrupts homeostasis of several cholesterol precursors, such as that of desmosterol, 7-DHC and 8-DHC, which translates into strong neurodegeneration impacting motor behavior in normal mice. Despite this, the levels of cholesterol remain unchanged. This is consistent with the observation that 24-OHC levels and cholesterol synthesis are decreased in aging brains, a finding that may explain why cholesterol content remains unmodified [69]. Moreover, in Thy-Tau 22 mice overexpressing CYP46A1, the levels of cholesterol remained unchanged in the mouse hippocampus while the levels of 24-OHC were increased [16]. Interestingly, while measuring total tissue cholesterol concentration did not reveal a significant variation in this very high cholesterol pool, modulation of the cholesterol content in lipid rafts fractions isolated from membranes could be evidenced in vitro and in vivo after CYP46A1 modulation. This suggests that, more than causing global modification of total cholesterol levels, CYP46A1 modulation could lead to a redistribution of cholesterol in cellular compartments. Further studies would be needed to validate this hypothesis.

The decreased expression of CYP46A1 in SCA3 patients and model mice brains prompted us to evaluate if long-lasting expression of CYP46A1 upon a single administration of AAV-CYP46A 1 to the brain region specifically affected by the disease in SCA3 mice could improve their phenotype. First, we used a lentiviral model in which the local expression of mutant human ataxin-3 is associated with strong nuclear aggregation and neurodegeneration $[4,48]$ and that has been used in the evaluation of several SCA3 disease modifiers $[2,46]$. We show that CYP46A1 expression concomitant to the expression of mutant ataxin-3 in the striatum significantly reduced the number and size of intranuclear protein aggregates, which are hallmarks of SCA3 and other polyQ disorders. This reduction was accompanied by the preservation of DARPP-32 immunoreactivity relatively to control animals injected with AAVrh10-GFP, emphasizing the neuroprotective effect conferred by CYP46A1.

To get further insight into the effects of CYP46A1 on motor behavior, and to show the ability of CYP46A1 administration to reverse an already established pathology, we turned to a transgenic SCA3 mouse model that expresses a truncated form ataxin-3 with a disease-associated polyQ tract. As reported for SCA3 patients [55, 60, 68], this mouse model exhibits pronounced cerebellar atrophy and significant PCs loss [53, 71], with consequent motor coordination deficits, such as gait ataxia. AAV-CYP46A1 injection was performed by 6 weeks of age, a time point in which cerebellar neuropathology and motor impairments are already very substantial $[53,71]$. Local intra-cerebellar expression of CYP46A1, in particular in PCs, triggered a significant amelioration of motor impairments of these severely affected SCA3 mice. Altogether, results in the lentiviral and the transgenic models of SCA3 serve as the proof-of-concept evidence that CYP46A1 expression can have both a preventive and curative effect in vivo, in mouse models of SCA3.

The cerebellum is not the only region of the nervous system affected in SCA3. Other brain regions, such as the striatum and substantia nigra, as well as some cranial nerve motor nuclei $[4,25,31,56]$ are widely associated with SCA3 neuropathology. Future translational steps towards a therapeutic approach for human patients will entail (a) the evaluation of broader delivery systems, to ensure efficient targeting of all affected regions, [22, 63]; (b) AAV serotypes able to cross the BBB and allowing for intravenous administration; (c) and improved delivery protocols (multi-sites or in cerebrospinal fluid), as previously described and used in preclinical/clinical trials [7, 34, 59, 73].

The impairment of autophagy has been evidenced in SCA3 [46, 51, 64] and in other polyQ diseases [20], while autophagy activation has been shown to counteract SCA3 phenotypes in mouse models. We here investigated the potential involvement of CYP46A1 and the cholesterol pathway in this mechanism $[21,45]$. Autophagy is an indispensable pathway in neurons, as the knockout of key autophagyrelated genes leads to accumulation of neuronal aggregates and neurodegeneration [27]. In this study we show that CYP46A1 silencing in the mouse brain induces strong accumulation of lysosomes and endosomes. Lysosome dysfunction has been linked to autophagolysosome accumulation [43]. Interestingly, we observed numerous lysosomes that appeared as electron-dense organelles containing 
osmiophilic material, therefore, suggesting the presence of undigested content. Similar data have been also observed in old Spg11 -/- mice [14]. Moreover, ultrastructural analysis of mice injected with shCYP46A1 in the hippocampus also revealed abnormal accumulation of lysosomes/autophagolysosomes with electron-dense vesicles [6] associated with endoplasmic reticulum stress and enlargement of the lysosomal compartment [24]. This compelling data suggest that autophagy could be implicated in CYP46A1 modulation of cholesterol metabolism. We thus analyzed, in vitro and in vivo, if the beneficial effect of CYP46A1 could be associated with restoration of the autophagic process. We demonstrated that CYP46A1 mediates clearance of both soluble and aggregated mutant ataxin-3 by an autophagymediated mechanism, as this effect was also observable upon UPS inhibition. This is in line with the observation that ataxin-3 degradation may be independent from ubiquitination and proteasome activity [12]. Besides, previous studies have clearly demonstrated that mutant ataxin-3 is degraded by autophagy [46]. CYP46A1 overexpression led to a significant increase of LC3B-II and expected reduction in p62/SQSTM1 levels, which are two important autophagy markers. Even more importantly, CYP46A1 led to a robust in vitro increase in the autophagic flux, which reflects an increase in the autophagic degradation activity. Moreover, CYP46A1 expression in the mouse brain was also able to activate autophagy, leading to an increase in LC3B-II levels and to a reduction in SQSTM1/p62 levels.

Results are in accordance with our previous observation that CYP46A1 delivery was able to markedly decrease polyQ huntingtin aggregates in a mouse model of HD [13]. Interestingly, this effect was also observed in the SCA2causative protein, as soluble and insoluble mutant ataxin-2 levels were significantly reduced upon CYP46A1 expression, thus showing a potentially beneficial effect for another polyQ disorder.

The current demonstration that CYP46A1 is decreased not only in patients and mouse models of $\mathrm{AD}$ [16] and $\mathrm{HD}$ [13] but also of polyQ-SCAs, together with the detection of impaired brain cholesterol synthesis, strongly argues for a central role of CYP46A1 and the cholesterol pathway in the physiopathology of these diseases. Results suggest that a CYP46A1 deficit could represent a direct causative factor in the neurodegenerative process. Based on these findings, we may hypothesize that cholesterol metabolism could be a critical and common path to neurodegeneration, regardless of the initiating mechanism. CYP46A1 has been identified as a stress response element of neurons and its expression was shown to be increased in early phases of degeneration [66]. The decreased expression of this enzyme during the evolution of the neurodegenerative process and its consequences on the production of key cellular precursors and on cellular pathways, particularly of autophagy, could lead to a toxic cascade and aggravate the detrimental consequences of aberrant protein accumulation and oxidative stress. This could contribute to the rapid exacerbation of cellular defects. Importantly, since our previous results show that this process is not limited to diseases with polyQ-protein accumulation-as the consequences of CYP46A1 expression or inhibition were demonstrated on amyloid disease $[24,28]$ and tauopathy [16] — this could represent a broader mechanism ensuring neuronal defense against toxic conditions. A key question that will need to be addressed is to better understand why and when the decreased expression of CYP46A1 begins during the course of specific pathologies.

Even if additional studies are needed to fill all these unanswered points, our data sturdily support that AAV-CYP46A1 gene therapy is an efficient approach to alleviate SCA3 progression, opening new avenues towards clinical applications targeting SCA3 and other SCAs.

Acknowledgements This work was supported by NeurATRIS: A Translational Research Infrastructure for Biotherapies in Neurosciences, the Fondation pour la Recherche Médicale, Bioingénierie pour la Santé 2014 "Project DBS20140930765", Paris Biotech Santé incubator, the SATT (Société d'Accélération de Transfert Technologique) Ile de France Innov, E.rare: E-Rare Joint Transnational Call for Proposals 2017 "Transnational Research Projects for Innovative Therapeutic Approaches for Rare Diseases", Biotheralliance network from the Paris Saclay University and Brainvectis. This work was also financed by the European Regional Development Fund (ERDF), through the CENTRO 2020 Regional Operational Programme under project CENTRO-01-0145-FEDER-000008:BrainHealth 2020, through the COMPETE 2020-Operational Programme for Competitiveness and Internationalization and Portuguese national funds via FCT-Fundação para a Ciência e a Tecnologia, I.P., under projects POCI-01-0145-FEDER-016719 (PTDC/NEU-NMC/0084/2014), POCI-01-0145-FEDER-007440 (UID/NEU/04539/2013) and POCI-01-0145-FEDER-016390:CANCEL STEM, and through CENTRO 2020 and FCT under project CENTRO-01-0145-FEDER022095:ViraVector; also by projects ESMI (JPCOFUND/0001/2015) and ModelPolyQ (JPCOFUND/0005/2015) under the EU Joint Program-Neurodegenerative Disease Research (JPND), the last two cofunded by the European Union H2020 program, GA No.643417 and national funds (FCT), and by the Richard Chin and Lily Lock Machado Joseph Disease Research Fund; and the National Ataxia Foundation. CN laboratory is supported by the French Muscular Dystrophy Association (AFM-Téléthon), the Ataxia UK, and the FCT. AM is supported by a Ph.D. fellowship from FCT (SFRH/BD/133192/2017).

\section{Compliance with ethical standards}

Conflict of interest The authors declare financial interest. CN, LM, NC, LPA and SA are inventors of patent applications claiming the use of AAV-CYP46A1 therapy in spinocerebellar ataxias.

\section{References}

1. Alves S, Cormier-Dequaire F, Marinello M, Marais T, Muriel MP, Beaumatin F et al (2014) The autophagy/lysosome pathway is impaired in SCA7 patients and SCA7 knock-in mice. Acta Neuropathol 128:705-722. https://doi.org/10.1007/s00401-014-1289-8 
2. Alves S, Nascimento-Ferreira I, Auregan G, Hassig R, Dufour N, Brouillet E et al (2008) Allele-specific RNA silencing of mutant ataxin-3 mediates neuroprotection in a rat model of MachadoJoseph disease. PLoS One 3:e3341. https://doi.org/10.1371/journ al.pone. 0003341

3. Alves S, Nascimento-Ferreira I, Dufour N, Hassig R, Auregan G, Nobrega $\mathrm{C}$ et al (2010) Silencing ataxin-3 mitigates degeneration in a rat model of Machado-Joseph disease: no role for wild-type ataxin-3? Hum Mol Genet 19:2380-2394. https://doi.org/10.1093/ $\mathrm{hmg} / \mathrm{ddq} 111$

4. Alves S, Regulier E, Nascimento-Ferreira I, Hassig R, Dufour $\mathrm{N}$, Koeppen A et al (2008) Striatal and nigral pathology in a lentiviral rat model of Machado-Joseph disease. Hum Mol Genet 17:2071-2083. https://doi.org/10.1093/hmg/ddn106

5. Aveleira CA, Botelho M, Carmo-Silva S, Pascoal JF (2015) Neuropeptide Y stimulates autophagy in hypothalamic neurons. Proc Natl Acad Sci 112:E1642-E1651. https://doi.org/10.1073/ pnas. 1416609112

6. Ayciriex S, Djelti F, Alves S, Regazzetti A, Gaudin M, Varin J et al (2017) Neuronal cholesterol accumulation induced by Cyp46a1 down-regulation in mouse hippocampus disrupts brain lipid homeostasis. Front Mol Neurosci 10:211. https://doi. org/10.3389/fnmol.2017.00211

7. Ayers JI, Fromholt S, Sinyavskaya O, Siemienski Z, Rosario AM, Li A et al (2015) Widespread and efficient transduction of spinal cord and brain following neonatal AAV injection and potential disease modifying effect in ALS mice. Mol Ther 23:53-62. https ://doi.org/10.1038/mt.2014.180

8. Bjorkhem I (2006) Crossing the barrier: oxysterols as cholesterol transporters and metabolic modulators in the brain. J Intern Med 260:493-508. https://doi.org/10.1111/j.1365-2796.2006.01725.x

9. Bjorkhem I, Leoni V, Meaney S (2010) Genetic connections between neurological disorders and cholesterol metabolism. J Lipid Res 51:2489-2503. https://doi.org/10.1194/jlr.R006338

10. Bjorkhem I, Lutjohann D, Diczfalusy U, Stahle L, Ahlborg G, Wahren J (1998) Cholesterol homeostasis in human brain: turnover of 24S-hydroxycholesterol and evidence for a cerebral origin of most of this oxysterol in the circulation. J Lipid Res 39:1594-1600

11. Bjorkhem I, Meaney S (2004) Brain cholesterol: long secret life behind a barrier. Arterioscler Thromb Vasc Biol 24:806-815. https://doi.org/10.1161/01.ATV.0000120374.59826.1b

12. Blount JR, Tsou WL, Ristic G, Burr AA, Ouyang M, Galante $\mathrm{H}$ et al (2014) Ubiquitin-binding site 2 of ataxin-3 prevents its proteasomal degradation by interacting with Rad23. Nat Commun 5:4638. https://doi.org/10.1038/ncomms5638

13. Boussicault L, Alves S, Lamaziere A, Planques A, Heck N, Moumne L et al (2016) CYP46A1, the rate-limiting enzyme for cholesterol degradation, is neuroprotective in Huntington's disease. Brain 139:953-970. https://doi.org/10.1093/brain/awv384

14. Branchu J, Boutry M, Sourd L, Depp M, Leone C, Corriger A et al (2017) Loss of spatacsin function alters lysosomal lipid clearance leading to upper and lower motor neuron degeneration. Neurobiol Dis 102:21-37. https://doi.org/10.1016/j.nbd.2017.02.007

15. Burk K, Fetter M, Abele M, Laccone F, Brice A, Dichgans J et al (1999) Autosomal dominant cerebellar ataxia type I: oculomotor abnormalities in families with SCA1, SCA2, and SCA3. J Neurol 246:789-797

16. Burlot MA, Braudeau J, Michaelsen-Preusse K, Potier B, Ayciriex S, Varin J et al (2015) Cholesterol 24-hydroxylase defect is implicated in memory impairments associated with Alzheimerlike Tau pathology. Hum Mol Genet 24:5965-5976. https://doi. org $/ 10.1093 / \mathrm{hmg} / \mathrm{ddv} 268$

17. Camargo N, Smit AB, Verheijen MH (2009) SREBPs: SREBP function in glia-neuron interactions. FEBS J 276:628-636. https ://doi.org/10.1111/j.1742-4658.2008.06808.x
18. Chai Y, Shao J, Miller VM, Williams A, Paulson HL (2002) Livecell imaging reveals divergent intracellular dynamics of polyglutamine disease proteins and supports a sequestration model of pathogenesis. Proc Natl Acad Sci USA 99:9310-9315. https:// doi.org/10.1073/pnas.152101299

19. Chevy F, Humbert L, Wolf C (2005) Sterol profiling of amniotic fluid: a routine method for the detection of distal cholesterol synthesis deficit. Prenat Diagn 25:1000-1006. https://doi. org/10.1002/pd.1254

20. Cortes CJ, La Spada AR (2015) Autophagy in polyglutamine disease: imposing order on disorder or contributing to the chaos? Mol Cell Neurosci 66:53-61. https://doi.org/10.1016/j. mcn.2015.03.010

21. Cunha-Santos J, Duarte-Neves J, Carmona V, Guarente L, Pereira de Almeida L, Cavadas C (2016) Caloric restriction blocks neuropathology and motor deficits in Machado-Joseph disease mouse models through SIRT1 pathway. Nat Commun 7:11445. https:// doi.org/10.1038/ncomms 11445

22. Deverman BE, Pravdo PL, Simpson BP, Kumar SR, Chan KY, Banerjee A et al (2016) Cre-dependent selection yields AAV variants for widespread gene transfer to the adult brain 34:204-209. https://doi.org/10.1038/nbt.3440

23. Dietschy JM, Turley SD (2002) Control of cholesterol turnover in the mouse. J Biol Chem 277:3801-3804. https://doi.org/10.1074/ jbc.R100057200

24. Djelti F, Braudeau J, Hudry E, Dhenain M, Varin J, Bieche I et al (2015) CYP46A1 inhibition, brain cholesterol accumulation and neurodegeneration pave the way for Alzheimer's disease. Brain 138:2383-2398. https://doi.org/10.1093/brain/awv166

25. Durr A, Stevanin G, Cancel G, Duyckaerts C, Abbas N, Didierjean O et al (1996) Spinocerebellar ataxia 3 and Machado-Joseph disease: clinical, molecular, and neuropathological features. Ann Neurol 39:490-499. https://doi.org/10.1002/ana.410390411

26. Dzeletovic S, Babiker A, Lund E, Diczfalusy U (1995) Time course of oxysterol formation during in vitro oxidation of low density lipoprotein. Chem Phys Lipid 78:119-128

27. Hara T, Nakamura K, Matsui M, Yamamoto A, Nakahara Y, Suzuki-Migishima R et al (2006) Suppression of basal autophagy in neural cells causes neurodegenerative disease in mice. Nature 441:885-889. https://doi.org/10.1038/nature04724

28. Hudry E, Van Dam D, Kulik W, De Deyn PP, Stet FS, Ahouansou $O$ et al (2010) Adeno-associated virus gene therapy with cholesterol 24-hydroxylase reduces the amyloid pathology before or after the onset of amyloid plaques in mouse models of Alzheimer's disease. Mol Ther 18:44-53. https://doi.org/10.1038/ mt.2009.175

29. Huynh DP, Yang HT, Vakharia H, Nguyen D, Pulst SM (2003) Expansion of the polyQ repeat in ataxin-2 alters its Golgi localization, disrupts the Golgi complex and causes cell death. Hum Mol Genet 12:1485-1496

30. Kawaguchi Y, Okamoto T, Taniwaki M, Aizawa M, Inoue M, Katayama S et al (1994) CAG expansions in a novel gene for Machado-Joseph disease at chromosome 14q32.1. Nat Genet 8:221-228. https://doi.org/10.1038/ng1194-221

31. Klockgether T, Skalej M, Wedekind D, Luft AR, Welte D, Schulz JB et al (1998) Autosomal dominant cerebellar ataxia type I. MRI-based volumetry of posterior fossa structures and basal ganglia in spinocerebellar ataxia types 1, 2 and 3. Brain 121(Pt 9): $1687-1693$

32. Korade Z, Kenworthy AK (2008) Lipid rafts, cholesterol, and the brain. Neuropharmacology 55:1265-1273. https://doi. org/10.1016/j.neuropharm.2008.02.019

33. Kotti TJ, Ramirez DM, Pfeiffer BE, Huber KM, Russell DW (2006) Brain cholesterol turnover required for geranylgeraniol production and learning in mice. Proc Natl Acad Sci USA 103:3869-3874. https://doi.org/10.1073/pnas.0600316103 
34. Lukashchuk V, Lewis KE, Coldicott I, Grierson AJ, Azzouz M (2016) AAV9-mediated central nervous system-targeted gene delivery via cisterna magna route in mice. Mol Ther Methods Clin Dev 3:15055. https://doi.org/10.1038/mtm.2015.55

35. Lund EG, Guileyardo JM, Russell DW (1999) cDNA cloning of cholesterol 24-hydroxylase, a mediator of cholesterol homeostasis in the brain. Proc Natl Acad Sci USA 96:7238-7243

36. Lund EG, Xie C, Kotti T, Turley SD, Dietschy JM, Russell DW (2003) Knockout of the cholesterol 24-hydroxylase gene in mice reveals a brain-specific mechanism of cholesterol turnover. J Biol Chem. https://doi.org/10.1074/jbc.M303415200

37. Margolis RL, Ross CA (2001) Expansion explosion: new clues to the pathogenesis of repeat expansion neurodegenerative diseases. Trends Mol Med 7:479-482

38. Martin MG, Pfrieger F, Dotti CG (2014) Cholesterol in brain disease: sometimes determinant and frequently implicated. EMBO Rep 15:1036-1052. https://doi.org/10.15252/embr.201439225

39. Matilla-Duenas A, Ashizawa T, Brice A, Magri S, McFarland KN, Pandolfo $M$ et al (2014) Consensus paper: pathological mechanisms underlying neurodegeneration in spinocerebellar ataxias. Cerebellum 13:269-302. https://doi.org/10.1007/s1231 1-013-0539-y

40. Mauch DH, Nagler K, Schumacher S, Goritz C, Muller EC, Otto A et al (2001) CNS synaptogenesis promoted by glia-derived cholesterol. Science 294:1354-1357. https://doi.org/10.1126/scien ce.294.5545.1354

41. Meaney S, Hassan M, Sakinis A, Lutjohann D, von Bergmann K, Wennmalm A et al (2001) Evidence that the major oxysterols in human circulation originate from distinct pools of cholesterol: a stable isotope study. J Lipid Res 42:70-78

42. Mendonca LS, Nobrega C, Hirai H, Kaspar BK, Pereira de Almeida L (2015) Transplantation of cerebellar neural stem cells improves motor coordination and neuropathology in MachadoJoseph disease mice. Brain 138:320-335. https://doi.org/10.1093/ brain/awu352

43. Menzies FM, Fleming A, Rubinsztein DC (2015) Compromised autophagy and neurodegenerative diseases. Nat Rev Neurosci 16:345-357. https://doi.org/10.1038/nrn3961

44. Moutinho M, Nunes MJ, Rodrigues E (2016) Cholesterol 24-hydroxylase: brain cholesterol metabolism and beyond. Biochem Biophys Acta 1861:1911-1920. https://doi.org/10.1016/j. bbalip.2016.09.011

45. Nascimento-Ferreira I, Nobrega C, Vasconcelos-Ferreira A, Onofre I, Albuquerque D, Aveleira C et al (2013) Beclin 1 mitigates motor and neuropathological deficits in genetic mouse models of Machado-Joseph disease. Brain 136:2173-2188. https://doi. org/10.1093/brain/awt144

46. Nascimento-Ferreira I, Santos-Ferreira T, Sousa-Ferreira L, Auregan G, Onofre I, Alves S et al (2011) Overexpression of the autophagic beclin-1 protein clears mutant ataxin-3 and alleviates Machado-Joseph disease. Brain 134:1400-1415. https://doi. org/10.1093/brain/awr047

47. Nobrega $C$, Carmo-Silva S, Albuquerque D, Vasconcelos-Ferreira A, Vijayakumar UG, Mendonca L et al (2015) Re-establishing ataxin-2 downregulates translation of mutant ataxin-3 and alleviates Machado-Joseph disease. Brain 138:3537-3554. https://doi. org/10.1093/brain/awv298

48. Nobrega C, Nascimento-Ferreira I, Onofre I, Albuquerque D, Conceicao M, Deglon N et al (2013) Overexpression of mutant ataxin-3 in mouse cerebellum induces ataxia and cerebellar neuropathology. Cerebellum 12:441-455. https://doi.org/10.1007/s1231 1-012-0432-0

49. Nobrega C, Nascimento-Ferreira I, Onofre I, Albuquerque D, Deglon N, de Almeida LP (2014) RNA interference mitigates motor and neuropathological deficits in a cerebellar mouse model of Machado-Joseph disease. PLoS One 9:e100086. https://doi. org/10.1371/journal.pone.0100086

50. Ohyama Y, Meaney S, Heverin M, Ekstrom L, Brafman A, Shafir $M$ et al (2006) Studies on the transcriptional regulation of cholesterol 24-hydroxylase (CYP46A1): marked insensitivity toward different regulatory axes. J Biol Chem 281:3810-3820. https://doi. org/10.1074/jbc.M505179200

51. Onofre I, Mendonca N, Lopes S, Nobre R, de Melo JB, Carreira IM et al (2016) Fibroblasts of Machado-Joseph disease patients reveal autophagy impairment. Sci Rep 6:28220. https://doi. org/10.1038/srep28220

52. Orr HT, Zoghbi HY (2007) Trinucleotide repeat disorders. Annu Rev Neurosci 30:575-621. https://doi.org/10.1146/annurev.neuro .29.051605.113042

53. Oue M, Mitsumura K, Torashima T, Koyama C, Yamaguchi $\mathrm{H}$, Furuya $\mathrm{N}$ et al (2009) Characterization of mutant mice that express polyglutamine in cerebellar Purkinje cells. Brain Res 1255:9-17. https://doi.org/10.1016/j.brainres.2008.12.014

54. Ramirez DM, Andersson S, Russell DW (2008) Neuronal expression and subcellular localization of cholesterol 24-hydroxylase in the mouse brain. J Comp Neurol 507:1676-1693. https://doi. org/10.1002/cne.21605

55. Reetz K, Costa AS, Mirzazade S, Lehmann A, Juzek A, Rakowicz $M$ et al (2013) Genotype-specific patterns of atrophy progression are more sensitive than clinical decline in SCA1, SCA3 and SCA6. Brain 136:905-917. https://doi.org/10.1093/brain/aws369

56. Rub U, Brunt ER, Deller T (2008) New insights into the pathoanatomy of spinocerebellar ataxia type 3 (Machado-Joseph disease). Curr Opin Neurol 21:111-116. https://doi.org/10.1097/ WCO.0b013e3282f7673d

57. Rub U, Brunt ER, Petrasch-Parwez E, Schols L, Theegarten D, Auburger $\mathrm{G}$ et al (2006) Degeneration of ingestion-related brainstem nuclei in spinocerebellar ataxia type 2, 3, 6 and 7. Neuropathol Appl Neurobiol 32:635-649. https://doi.org/10.111 1/j.1365-2990.2006.00772.x

58. Russell DW, Halford RW, Ramirez DM, Shah R, Kotti T (2009) Cholesterol 24-hydroxylase: an enzyme of cholesterol turnover in the brain. Annu Rev Biochem 78:1017-1040. https://doi. org/10.1146/annurev.biochem.78.072407.103859

59. Samaranch L, Salegio EA, San Sebastian W, Kells AP, Foust KD, Bringas JR et al (2012) Adeno-associated virus serotype 9 transduction in the central nervous system of nonhuman primates. Hum Gene Ther 23:382-389. https://doi.org/10.1089/hum.2011.200

60. Scherzed W, Brunt ER, Heinsen H, de Vos RA, Seidel K, Burk $\mathrm{K}$ et al (2012) Pathoanatomy of cerebellar degeneration in spinocerebellar ataxia type 2 (SCA2) and type 3 (SCA3). Cerebellum 11:749-760. https://doi.org/10.1007/s12311-011-0340-8

61. Schols L, Amoiridis G, Buttner T, Przuntek H, Epplen JT, Riess O (1997) Autosomal dominant cerebellar ataxia: phenotypic differences in genetically defined subtypes? Ann Neurol 42:924-932. https://doi.org/10.1002/ana.410420615

62. Schols L, Bauer P, Schmidt T, Schulte T, Riess O (2004) Autosomal dominant cerebellar ataxias: clinical features, genetics, and pathogenesis. Lancet Neurol 3:291-304. https://doi.org/10.1016/ S1474-4422(04)00737-9

63. Schuster DJ, Dykstra JA, Riedl MS, Kitto KF, Belur LR, McIvor RS et al (2014) Biodistribution of adeno-associated virus serotype 9 (AAV9) vector after intrathecal and intravenous delivery in mouse. Front Neuroanat 8:42. https://doi.org/10.3389/fnana .2014 .00042

64. Sittler A, Muriel MP, Marinello M, Brice A, den Dunnen W, Alves S (2017) Deregulation of autophagy in postmortem brains of Machado-Joseph disease patients. Neuropathology. https://doi. org/10.1111/neup. 12433

65. Sobo K, Le Blanc I, Luyet PP, Fivaz M, Ferguson C, Parton RG et al (2007) Late endosomal cholesterol accumulation leads to 
impaired intra-endosomal trafficking. PLoS One 2:e851. https:// doi.org/10.1371/journal.pone.0000851

66. Sodero AO, Trovo L, Iannilli F, Van Veldhoven P, Dotti CG, Martin MG (2011) Regulation of tyrosine kinase B activity by the Cyp46/cholesterol loss pathway in mature hippocampal neurons: relevance for neuronal survival under stress and in aging. J Neurochem 116:747-755. https://doi.org/10.111 1/j.1471-4159.2010.07079.x

67. Sodero AO, Vriens J, Ghosh D, Stegner D, Brachet A, Pallotto M et al (2012) Cholesterol loss during glutamate-mediated excitotoxicity. EMBO J 31:1764-1773. https://doi.org/10.1038/emboj .2012 .31

68. Stefanescu MR, Dohnalek M, Maderwald S, Thurling M, Minnerop M, Beck A et al (2015) Structural and functional MRI abnormalities of cerebellar cortex and nuclei in SCA3, SCA6 and Friedreich's ataxia. Brain 138:1182-1197. https://doi.org/10.1093/ brain/awv064

69. Thelen KM, Falkai P, Bayer TA, Lutjohann D (2006) Cholesterol synthesis rate in human hippocampus declines with aging. Neurosci Lett 403:15-19. https://doi.org/10.1016/j.neulet.2006.04.034

70. Toonen LJA, Overzier M, Evers MM, Leon LG, van der Zeeuw SAJ, Mei H et al (2018) Transcriptional profiling and biomarker identification reveal tissue specific effects of expanded ataxin-3 in a spinocerebellar ataxia type 3 mouse model. Mol Neurodegener 13:31. https://doi.org/10.1186/s13024-018-0261-9

71. Torashima T, Koyama C, Iizuka A, Mitsumura K, Takayama K, Yanagi $S$ et al (2008) Lentivector-mediated rescue from cerebellar ataxia in a mouse model of spinocerebellar ataxia. EMBO Rep 9:393-399. https://doi.org/10.1038/embor.2008.31

72. Vance JE (2012) Dysregulation of cholesterol balance in the brain: contribution to neurodegenerative diseases. Dis Models Mech 5:746-755. https://doi.org/10.1242/dmm.010124

73. Zerah M, Piguet F, Colle MA, Raoul S, Deschamps JY, Deniaud J et al (2015) Intracerebral gene therapy using AAVrh. 10-hARSA recombinant vector to treat patients with early-onset forms of metachromatic leukodystrophy: preclinical feasibility and safety assessments in nonhuman primates. Hum Gene Ther Clin Dev 26:113-124. https://doi.org/10.1089/humc.2014.139

Publisher's Note Springer Nature remains neutral with regard to jurisdictional claims in published maps and institutional affiliations.

\section{Affiliations}

\section{Clévio Nóbrega ${ }^{1,2,3,4} \cdot$ Liliana Mendonça $^{4} \cdot$ Adriana Marcelo $^{1,2} \cdot$ Antonin Lamazière $^{5} \cdot$ Sandra Tomé ${ }^{4}$. Gaetan Despres ${ }^{5} \cdot$ Carlos A. Matos $^{1,2,4} \cdot$ Fatich Mechmet $^{1,2}$. Dominique Langui ${ }^{6}$. Wilfred den Dunnen ${ }^{7}$. Luis Pereira de Almeida ${ }^{4,10} \cdot$ Nathalie Cartier ${ }^{8,9} \cdot$ Sandro Alves $^{11}$ (i)}

Luis Pereira de Almeida luispa@cnc.uc.pt

$\triangle$ Nathalie Cartier nathalie.cartier@inserm.fr

Sandro Alves sandro.alves@brainvectis.com; sandropfalves@gmail.com

1 Department of Biomedical Sciences and Medicine, University of Algarve, Faro, Portugal

2 Centre for Biomedical Research, University of Algarve, Faro, Portugal

3 Algarve Biomedical Center, University of Algarve, Faro, Portugal

4 Center for Neuroscience and Cell Biology, University of Coimbra, Coimbra, Portugal

5 INSERM, Saint-Antoine Research Center, Sorbonne Université, Faculté de Médecine, AP-HP, Hôpital Saint Antoine, Département PM2, Paris, France
6 Institut du Cerveau et de la Moelle épinière, ICM, INSERM U1127, CNRS UMR7225, Sorbonne Université, Hôpital Pitié-Salpêtrière, 47 bd de l'Hôpital, 75013 Paris, France

7 Department of Pathology and Medical Biology, University Medical Center Groningen, University of Groningen, PO Box 30.001, 9700 RB Groningen, The Netherlands

8 INSERM U1169 92265 Fontenay aux Roses and Université Paris-Sud, Université Paris Saclay, 91400 Orsay, France

9 Present Address: INSERM U1127, Institut du Cerveau et de la Moelle épinière (ICM), Hôpital Pitié-Salpêtrière, 47 bd de l'hôpital, 75013 Paris, France

10 Faculty of Pharmacy, University of Coimbra, 3000-548 Coimbra, Portugal

11 Brainvectis, Institut du Cerveau et de la Moelle épinière (ICM), Hôpital Pitié-Salpêtrière, 47 boulevard de l'Hôpital Paris, 75646 Paris, CEDEX 13, France 